The University of San Francisco

USF Scholarship: a digital repository @ Gleeson Library |

Geschke Center

1989

\title{
Inverse Ac Josephson Effect at Terahertz Frequencies
}

\author{
W C. Danchi \\ William Golightly \\ University of San Francisco, wjgolightly@usfca.edu \\ E C. Sutton
}

Follow this and additional works at: http://repository.usfca.edu/phys

Part of the Physics Commons

\section{Recommended Citation}

W. C. Danchi, W. J. Golightly and E. C. Sutton. Inverse Ac Josephson Effect at Terahertz Frequencies. J. Appl. Phys. 65, 2772 (1989). 


\section{A P J Journal of}

\section{Inverse ac Josephson effect at terahertz frequencies}

W. C. Danchi, W. J. Golightly, and E. C. Sutton

Citation: Journal of Applied Physics 65, 2772 (1989); doi: 10.1063/1.342768

View online: http://dx.doi.org/10.1063/1.342768

View Table of Contents: http://scitation.aip.org/content/aip/journal/jap/65/7?ver=pdfcov

Published by the AIP Publishing

\section{Articles you may be interested in}

Inversion of complex $\mathrm{V}(\mathrm{z})$ at high frequencies for acoustic microscopy

Rev. Sci. Instrum. 67, 2656 (1996); 10.1063/1.1147187

Critical radius, size effects and inverse problems for composites with imperfect interface

J. Appl. Phys. 79, 8964 (1996); 10.1063/1.362628

Soliton return effect in an annular Josephson junction and nonzero minimum voltage and current in the current step

J. Appl. Phys. 71, 1014 (1992); 10.1063/1.350437

Theoretical investigations of microwave-driven Josephson junctions in a large frequency range J. Appl. Phys. 66, 735 (1989); 10.1063/1.343547

Josephson effect above $77 \mathrm{~K}$ in a YBaCuO break junction

Appl. Phys. Lett. 51, 540 (1987); 10.1063/1.98392

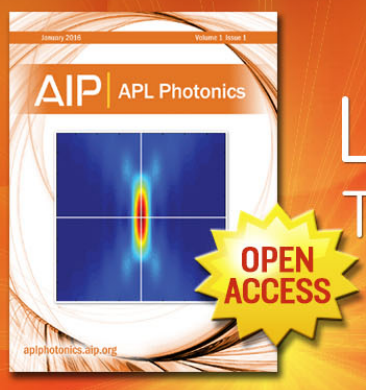




\title{
Inverse ac Josephson effect at terahertz frequencies
}

\author{
W. C. Danchi, W. J. Golightiy, and E. C. Sutton \\ Department of Physics and Space Sciences Laboratory, University of California, Berkeley, Califomia 94720
}

(Received 16 August 1988; accepted for publication 22 November 1988)

\begin{abstract}
The inverse ac Josephson effect occurs when a Josephson junction driven by a microwave source of frequency $f$ produces constant-voltage steps at integer multiples of $h f / 2 e$. For lowleakage current hysteretic junctions driven at microwave frequencies below about $100 \mathrm{GHz}$, some of these steps can cross the zero de bias current axis. These zero-crossing steps allow modern series array voltage standards to operate without individually biasing the junctions in the array. We reexamine the theory behind these steps and show that they can exist at frequencies much higher than thought previously. The Riedei singularity in the supercurrent response allows this effect to exist even up to terahertz frequencies. We describe a set of analytical calculations which provide limits on the amount of rounding of the Riedel peak which can be permitted while still allowing these zero-crossing steps to occur. We also discuss practical considerations such as microwave power levels required and parameters for device fabrication. This analysis is supported by numerical frequency-domain computations and timedomain simulations for a number of realistic $L-V$ curves with rounded Riedel singularities and with quasiparticle subgap leakage currents.
\end{abstract}

\section{INTRODUCTION}

The inverse ac Josephson effect is the occurrence of quantized de voltages $V_{n}=n \hbar \omega / 2 e$ for a Josephson junction driven by a microwave source. ${ }^{1}$ Levinsen $e t a l^{2}$ have shown that for hysteretic junctions constant-voltage steps car cross the zero-current axis. In this paper we will refer to these steps as zero-crossing steps. Kautz ${ }^{3}$ and Niemeyer, Hinken, and $\mathrm{Kaut}^{4}$ have shown experimentally that zero-crossing steps can be used with series arrays of 1474 junctions at 90 GHz to produce voltages as high as $1.2 \mathrm{~V}$. More recently the National Bureau of Standards 5 has made voltage standards of up to $10 \mathrm{~V}$ using series arrays of up to 14184 junctions. From the usual theoretical point of view, that of the StewartMcCumber model, ${ }^{6}$ also known as the resistively shunted junction (RSJ) nodel, it is expected that zero-crossing steps can exist only at voltages (or frequencies) up to about onehalf the energy gap of the superconducting tunnel junction. ${ }^{3}$ Experimental work by Hamilton, ${ }^{7}$ McDonald et al., Weitz, Skocpol, and Tinkham, ${ }^{9}$ Danchi, Habbal, and Tinkham, ${ }^{10}$ and Habbal, Danchi, and Tinkham, "shows that the simple RSJ model of the tunnel junction is not appropriate at high frequencies. Rather one must use the full theory of the junction, as developed by Werthamer, ${ }^{12}$ for understanding their behavior at such frequencies. This theory includes two important effects left out of the Stewart-McCumber model: the presence of photon-assisted quasiparticle tunneling steps discovered experimentally by Dayem and Martin, ${ }^{13}$ and explained by Tien and Gordon, ${ }^{14}$ and the Riedel peak ${ }^{15}$ in the supercurrent response.

In this work we show from the Werthamer theory of the superconducting tunnel junction that zero-crossing ac Josephson steps can occur at frequencies much higher than thought possibie previously. We begin with a review of the conventional picture of the Josephson junction voltage standard ${ }^{3}$ derived from the RSI model in Secs. II $A$ and II $B$. Following this we review the Werthamer theory ${ }^{12}$ of the $J_{0}$ sephson junction in Sec. II C. This review allows us to devel- op the tools necessary for our picture of zero-crossing steps at high frequencies, which is the main thrust of this work. Readers who are knowledgeable about the RSJ model and Werthamer theory may wish to skip over Secs. II A-II C. In Sec. II D we analyze the condition for the zero crossing of the Josephson steps based on the Werthamer picture in the frequency domain. This treatment is novel in that it takes into account the rounding of the Riedel peak and the presence of quasiparticle photon-assisted tunneling steps, for a junction driven by a microwave voltage source. Next, in Sec. II $\mathrm{E}$, we extend this analysis to an ac current source by finding the equivalent voltage source for a particular shunt capacitance. This unites the conventional low-frequency RSJ expressions with expressions that do not rely on any lowfrequency assumptions. We describe some of the practical considerations involved in experimental verification of this work in Sec. III. In Sec. IV A we report numerical computations based on the voltage-bias theory of Secs. II D and II E. Preliminary results from numerical simulations based on Harris's ${ }^{16,17}$ time-domain formulation of the Werthamer theory are discussed in Secs. IV B and IV C. These simuiations support the analytical calculations derived earlier.

\section{H. INVERSE AC JOSEPHSON EFFECT}

\section{A. RSJ picture}

The simplest and most commonly used picture in the analysis of the behavior of the Josephson junction is the Stewart-McCumber model ${ }^{t}$ or RSJ model. In this model the Josephson junction is represented by the parallel combination of a resistor $R$ to approximate the flow of quasiparticle currents, a capacitor $C$, and a current source of magnitude $I_{C} \sin \phi$ to represent the fow of pair currents. Here $\phi$ is the phase difference across the junction and $I_{C}$ is the critical current. In the ideal zero-temperature case the product of $I_{C}$ and the normal state resistance $R_{N}$ is given by

$$
I_{C} R_{N}=\pi \Delta(0) / 2 e .
$$


The Josephson relation,'

$$
V=\hbar \dot{\phi} / 2 e,
$$

connects the voltage across the junction to the derivative of the phase difference with respect to time.

If the junction is driven by the combination of a de current source $I_{0}$ and microwave source of magnitude $I_{1}$ at frequency $\omega_{L}$, then the differential equation describing the time evolution of the voltage across the junction is

$$
C \frac{d V}{d t}+\frac{V}{R}+I_{C} \sin \phi=I_{0}+I_{1} \cos \omega_{L} t
$$

where this equation is derived from current conservation. For ease of mathematical analysis it is conventional to normalize currents to the critical current, voltages to the $I_{C} R$ product, and angular frequencies to $2 e F_{C} R / h$. Typically $R$ is interpreted as the quasiparticle leakage resistance $R_{L}$ for voltages below the gap voltage $2 \Delta / e$ and as the normal state resistance $R_{N}$ for voltages above the gap voltsge. Therefore, we introduce the normalized quantities

$$
\begin{aligned}
& i_{0}=I_{0} / I_{C}, \quad i_{1}=I_{1} / I_{C}, \\
& v=V / I_{C} R, \quad \Omega=\omega_{L}\left(h / 2 e I_{C} R\right), \\
& \tau=t\left(2 e I_{C} R / h\right) .
\end{aligned}
$$

The Josephson relation (2) is rewritten as

$$
\frac{d \phi}{d \tau}=\nu=\dot{\phi}
$$

The equation of motion for the junction phase after some manipulation of Eq. (3) is

$$
\beta \ddot{\phi}+\dot{\phi}+\sin \phi=i_{0}+i_{1} \cos \Omega \tau \text {. }
$$

The quantity $\beta$ is

$$
\beta=2 e R_{C} R^{2} C / \text {, }
$$

and the derivatives with respect to normalized time $\tau$ are denoted by the dots over $\phi$. This $\beta$ is the well-known McCumber parameter ${ }^{6}$ describing the damping in the junction. In the limit where the phase excursions are small, the junction has a resonant response at a frequency $\Omega=\beta^{-1 / 2}$ with quality factor $Q \sim \beta^{1 / 2}$. This resonant frequency is also called the plasma frequency and is written

$$
\omega_{p}=\left(2 e I_{C} / \hbar C\right)^{1 / 2}
$$

in conventional units. In the linearized picture of the junction, the Josephson element appears as an inductor $L$, with inductance

$$
L_{J}=\hbar / 2 a I_{C}
$$

which can be derived from Eqs. (2) and (3) in the limit of small phase excursions. In this case the current throtigh the Josephson element is proportional to the time integral of the voltage, as with any inductor:

$$
I_{J}=\frac{2 e l_{c}}{n} \int V d t
$$

When a junction is driven by a microwave source of frequency $\omega_{L}$, the junction phase can be locked to the phase of the source, resulting in ac current steps at voltages $V_{n}=n\left(\hbar \omega_{L} / 2 e\right)$ for integer values of $n$. In the simplest case, the junction can be viewed as driven by a dc voltage $V_{0}$ and if voltage $V_{L} \cos \omega_{L} t$. If the junction has zero capacitance, and if it is driven by voltage sources only $\left(E_{0}=I_{1}=0\right.$ ), then from Eq. (3), as shown first by Shapiro, Janus, and Holly, ${ }^{1}$ the time-averaged or de current in the junction is given by

$$
\begin{aligned}
\langle I\rangle & =\left(V_{0} / R\right\rangle+I_{C}{ }_{-n}(2 \alpha) \sin \left(\phi_{0}\right) \text { if } V_{0}=n h_{\omega_{L}} / 2 e \\
& =V_{0} / R \text { otherwise. }
\end{aligned}
$$

In this equation $\alpha \equiv e V_{i} / w_{L}, J_{n}$ is the $n$ th-order Bessei function and $\phi_{0}$ is the initial junction phase. By varying the phase $\phi_{0}$, one finds that the step extends from $-I_{C} J_{n}(2 \alpha)$ to $+I_{C} I_{n}(2 a)$ from the ohmic line $V_{0} / R$.

A somewhat more complicated situation occurs when the Josephson junction is driven by dc and ac current sources as in Eqs. (3) or (6). If the reactance of the Josephson element $\omega_{L} L_{j}$ is much greater than the resistance $R$ or the capacitive reactance $1 / \omega_{I} C$, then most of the current passes through the capacitor or resistor, and it can be shown that the Josephson steps have a Bessel function dependence. ${ }^{3}$ If the drive current is large in comparison to the current through the Josephson element, Bessel function amplitudes have been shown to occur as well. ${ }^{3}$ In nomalized form these conditions are written ${ }^{3}$

$$
\begin{aligned}
& \Omega \gg 1, \\
& \Omega \gg \beta^{-i / 2}, \\
& i_{1} \gg 1 .
\end{aligned}
$$

In this limit one can derive ${ }^{3,18}$ from Eq. (6) an equation for the junction voltage $v=\dot{\phi}$,

$v=\langle v\rangle+\left(i_{1} / \sqrt{1}+\bar{\Omega}^{2} \beta^{2}\right) \cos \left(\Omega \tau+\phi_{a}\right)$,

and by integration one can obtain the junction phase

$\phi=\phi_{0}+\langle v\rangle \tau+\left(i_{1} / \Omega \sqrt{1}+\bar{\Omega}^{2} \bar{\beta}^{2}\right) \sin \left(\Omega \tau+\phi_{d}\right)$

where

$$
\phi_{\alpha}=\tan ^{-1}(-\Omega \beta)
$$

is the phase of the voltage relative to the phase of the drive current. If we define

$$
\tilde{i}_{1}=i_{1} / \Omega \sqrt{1+\Omega^{2}} \bar{\beta}^{2} \text {, }
$$

then the current through the junction is given by

$$
\begin{aligned}
i_{J} & =\sin \phi \\
& =\sum_{n=-\infty}^{\infty} J_{-n}\left(\tilde{i}_{R}\right) \sin \left[\phi_{0}-n \phi_{d}+(\langle\nu\rangle-n \Omega) \tau\right] .
\end{aligned}
$$

From Eqs. (15) and (6) we obtain an equation analogous to that of (10) for the time-averaged current through the junction when the junction is locked to the drive frequency

$$
\begin{aligned}
\langle v\rangle= & n \Omega, \\
i_{0} & =\langle v\rangle+\left\langle i_{j}\right\rangle \\
& =\langle v\rangle+J_{\ldots n}\left(\tilde{i}_{1}\right) \sin \left(\phi_{0}-n \phi_{d}\right),
\end{aligned}
$$

otherwise when $\langle v\rangle \neq n \Omega$ then $\langle v\rangle=i_{0}$. Therefore, the Josephson steps occur at voltages $\langle v\rangle=n \Omega$ and extend from $+J_{n}\left(\tilde{i}_{1}\right)$ to $-J_{n}\left(\tilde{i}_{1}\right)$, or in non-nomalized units they extend from $+I_{C} J_{n}\left(\tilde{i}_{1}\right)$ to $-I_{C} J_{n}\left(\tilde{i}_{i}\right)$. We observe also that there is a relation between the equivalent microwave voltage 
across the junction and the normalized drive current given by

$$
2 \alpha=\tilde{i}_{1} .
$$

\section{B. Zero-crossing steps in the RSI picture}

In the RSJ picture, as has been noted by Levinsen et al.? constant-voltage Josephson steps cross the zero-current axis when $i_{0}=0$, or from Eq. (16) when

$$
n \Omega<\left|\mathscr{J}_{n}(\tilde{i})\right|,
$$

where we have used the fact that the voltage on the Josephson step $\langle v\rangle=n \Omega$. Because $J_{n}$ is less than 0.58 for $n \geqslant 1, \mathbb{E}$. (18) suggests that there can be zero-crossing steps only for step voltages $V_{\text {step }}=\hbar \omega_{L} / 2 e$ given by

$$
V_{\text {step }}<0.46 V_{\text {gap }}\left(R / R_{N}\right) \text {, }
$$

where we have taken $n=1, V_{\text {gap }}=2 \Delta / e$, and we have converted from normalized to conventional notation. We assume that $R>R_{N}$ below the gap voltage and $R \sim R_{N}$ above the gap. For junctions with realistic $I-V$ curves in which $R / R_{N} \sim 5-10$, Eq. (19) states that one can in principle have Josephson steps which cross the zero axis for voltages all the way up to the gap voltage. Above the gap voltage, $V_{\text {step }}>V_{\text {gap }}=2 \Delta / e$ and the resistance $R \sim R_{N}$ which produces a contradiction of Eq. (19); thus the zero-crossing condition cannot be satisfied. However, the possibility still remains that zero-crossing steps could exisi to voltages nearIy equal to the gap voltage. It may be difficult to produce steps at voltages this high, because within the context of the RSJ model there is a conflict between the conditions for the zero-crossing steps and the conditions for Bessel function step amplitudes. For example, the condition $\Omega \gg 1$ is clearly inconsistent with zero-crossing steps [cf. Eq. (18)]. The second condition states that $\Omega^{2} B>1$, so that if $\Omega<1$ then $\beta \gg 1$ and thus the junction must be quite hysteretic. Another problem, first pointed out by Kaut,$^{3}$ is that near the gap voltage the quasiparticle photon-assisted tunneling steps move the quasiparticle I-V curve away from the current axis. We conclude that the RSJ model indicates that it may be difficult to obtain zero-crossing steps anywhere except at low frequencies and low voltages where the quasiparticle $I-V$ curve is close enough to the zero-current axis for the zerocrossing condition to be fulfilled for relatively small step amplitudes.

We shall show in Sec. II D that these conditions are not supported by an analysis within the framework of the Werthamer theory of the superconducting tunnel junction. We provide a capsule summary of the main aspects of the Werthamer theory and then analyze the zero-crossing behavior.

\section{Werthamer picture}

Some time ago Werthamner ${ }^{2}$ derived expressions for the tunneling current given a time-dependent voltage source $V(t)=V_{0}+\hat{V}(t)$ where $V_{0}$ contains the entire de component. His expression includes both quasiparticle and pair tunneling in a natural way developed from the tunneling hamiltonian formalism. Harris ${ }^{16,17}$ developed a time-domain formulation of the tumeling current derived from a slightly different form of Werthamer's frequency-domain expression. In order to maintain consistency with Harris and with other workers when we discuss numerical simulations done in the time domain, we begin our presentation with an expression equivalent to Harris's ${ }^{16}$ Eq. (1) where we follow Werthamer's notation in scparating the de from the timedependent voltage

$$
\begin{aligned}
I(t)= & \operatorname{Im} \int_{\approx}^{\infty} d \omega d \omega^{\prime} \\
& \times\left[W^{*}(\omega) W\left(\omega^{\prime}\right) e^{i\left(\omega^{\prime}-\omega^{\prime}\right)} I_{a p}\left(\omega+\frac{1}{2} \omega_{0}\right)\right. \\
& \left.+W(\omega) W\left(\omega^{\prime}\right) e^{\cdots i\left(\omega+\omega^{\prime}\right) t-i \omega_{0} t+i \alpha_{0}} I_{j}\left(\omega+\frac{1}{2} \omega_{0}\right)\right] .
\end{aligned}
$$

The Fourier transform of $W(\omega)$ is related to the voltage $V(t)$ by the following expression:

$\exp \left(\frac{-i e}{\hbar} \int_{-\infty}^{t} \hat{V}\left(t^{\prime}\right) d t^{\prime}\right)=\int_{-\infty}^{\infty} d \omega W(\omega) e^{-i \omega t}$.

We define the frequency $\omega_{0}$ to be $\omega_{0} \equiv 2 e V_{0} / \hbar$ while we define the phase $\alpha_{0}$ to be the initial phase diference.

The functions $l_{\text {up }}$ and $l_{J}$ are the complex-valued response functions in the frequency domain. $I_{\mathrm{C}}(\omega)$ describes the response of the quasiparticles to a frequency $\omega$, and $I_{f}(\omega)$ describes the response of the pair currents. The total current through the junction is the sum of these two contributions. General expressions can be found for these response functions that include both finite (nonzero) temperature as well as quasiparticle lifetime and gap anisotropy effects. ${ }^{19,20}$ In the limit when $T=0$, Werthamer ${ }^{12}$ derived analytical expressions for these response functions. Here we display expressions for the case of identical gaps on both sides of the junction, $\Delta_{1}=\Delta_{2}=\Delta$, which we derived from Werthamer's Eq. (13):

$$
\begin{aligned}
& I_{q p}(\omega)=\frac{\Delta}{e R_{N}}\left(K(x)-2 E(x)+\frac{\pi}{2}\right) \quad x \leqslant 1 \\
& =\frac{\Delta}{e R_{N}}\left(\int 2 x\left[K\left(\frac{1}{x}\right)-E\left(\frac{1}{x}\right)\right]-\frac{1}{x} K\left(\frac{1}{x}\right)\right. \\
& \left.+\frac{\pi}{2}\right\}+i \operatorname{sgn} \omega\left[2 x E\left[\left(1-\frac{1}{x^{2}}\right)^{1 / 2}\right]\right. \\
& \left.\left.-\frac{1}{x} K\left[\left(1-\frac{1}{x^{2}}\right)^{1 / 2}\right]\right]\right) x \geqslant 1 \text {, } \\
& I_{J}(\omega)=-\frac{\Delta}{e R_{N}} K(x) \quad x \leqslant 1 \\
& =-\frac{\Delta}{e R_{N}} \frac{1}{x}\left\{K\left(\frac{1}{x}\right)+i \operatorname{sgn} \omega K\left[\left(1-\frac{1}{x^{2}}\right)^{1 / 2}\right]\right\} \\
& x \geqslant 1 \text {. }
\end{aligned}
$$

In these expressions, $x \equiv=\hbar / 2 \Delta \mid, K$ and $E$ are complete elliptic integrals of the first and second kind, respectively, ans $\operatorname{sgn} \omega=+1$ for $\omega>0$ and $\operatorname{sgn} \omega=-1$ for $\omega<0$. These functions can be understood physically by assuming the voltage source has only a dc component, $V(t)=V_{0}$. Equation (21) implics that $W(\omega)=\delta(\omega)$ under this circumstance. If this $W(\omega)$ is inserted into Eq. (20) and the resulting expression is integrated, then it can be shown that 
the de $I-V$ curve of the junction is related to the imaginary part of the quasiparticle response function, i.e., $I_{\mathrm{dc}}\left(V_{0}\right)=\operatorname{Im}\left[I_{\mathrm{qp}}\left(V_{0}\right)\right]$ when $V_{0}$ is substituted for ho/e. Similarly, the critical current $l_{C}$ of the RSJ model is related to the real part of the Josephson response function in the frequency domain by $I_{C} \approx \operatorname{Re}\left[I_{3}\left(e V_{0} / h\right)\right]$ for $V_{0} \ll 2 \Delta / e$.

The real and imaginary parts of the response functions are related by the Kramers-Kronig transiorm, as discussed by Harris, ${ }^{21}$ i.e., if we write $I_{\mathrm{qp}}=I_{\mathrm{qp} 1}+I_{\mathrm{qp} 2}$ and $I_{J}=I_{J 1}+i I_{J 2}$, then

$$
\begin{aligned}
& I_{\mathrm{qp} 1}(\omega)=\frac{2}{\pi} \mathrm{P} \int_{0}^{\infty} \frac{\omega^{\prime} l_{\mathrm{qp} 2}\left(\omega^{\prime}\right) d \omega^{\prime}}{\omega^{\prime 2}-\omega^{2}}, \\
& I_{\mathrm{qp} 2}(\omega)=\frac{2 \omega}{\pi} \mathrm{P} \int_{0}^{\infty} \frac{I_{\mathrm{qp} 1}\left(\omega^{\prime}\right) d \omega^{\prime}}{\omega^{2}-\omega^{\prime 2}},
\end{aligned}
$$

and similarly for $I_{J 1}$ and $I_{J 2}$. Here $P$ denotes the principal vaiue of the integrals. Because the real and imaginary parts of the frequency-domain response functions are related by Kramers-Kronig transforms, the time-domain response functions $I_{j}(t)$ and $I_{\mathrm{qp}}(t)$ must be real and causal, i.e., that $I_{J}(t)=I_{\mathrm{qp}}(t)=0$ for $t<0$. The quasiparticle time-domain response function $I_{q p}(t)$ can be found by Fourier transforming the requency-domain expression $I_{\mathrm{ap}}(\omega)$ :

$$
\begin{aligned}
I_{\mathrm{qp}}(t) & =\int_{\infty}^{\infty} I_{\mathrm{qp}}(\omega) e^{-i \omega t} d \omega \\
& =\int_{-\infty}^{\infty}\left[I_{\mathrm{qp} 1}(\omega)+i I_{\mathrm{qp} 2}(\omega)\right] e^{-i \omega t} d \omega,
\end{aligned}
$$

and similarly for $I_{J}(t)$. The reality and causality of $I_{\mathrm{ap}}(t)$ is evident if we insert Eq. (24) into this equation and perform the indicated contour integration in $\omega^{\prime}$.

We wish to compare the predictions of the Werthamer theory with those of the simpler RSI model, in particular the effect of the Riedel peak. In deriving the step amplitudes we follow a procedure similar to that used earlier for the RSJ model. We assume that the sinusoidal driving voltage is of the form

$$
V(t)=V_{0}+V_{L} \cos \omega_{L} t
$$

We derive from this voltage and Eq. (21) the function $W(\omega)$, which describes the Fourier components (harmonics) of the driving voltage which affect the evolution of the junction

$$
W(\omega)=\sum_{k=-\infty}^{\infty} J_{k}\left(\frac{e V_{L}}{\hbar \omega_{L}}\right) \delta\left(\omega-k \omega_{L}\right) .
$$

This equation can be inserted into Eq. (20), and if we defire $a \equiv e V_{L} / \hbar \omega_{L}$, then we obtain for $I(t)$

$$
\begin{aligned}
& I(t)=\operatorname{Im}\left(\sum_{k_{s} k^{\prime}}^{\infty} \sum_{=\infty} J_{k}(\alpha) J_{k^{\prime}}(\alpha) e^{\cdots i\left(k-k^{\prime}\right) \omega_{L^{k}}}\right. \\
& \times I_{\mathrm{qp}}\left(k \omega_{L}+\frac{1}{2} \omega_{0}\right)+\sum_{k, k}^{\infty} \sum_{-\infty}^{\infty} J_{k}(\alpha) \\
& \left.\times B_{k^{\prime}}(\alpha) e^{-i\left(k+k^{\prime}\right) \omega_{L} t-i \omega_{0}, r+i \alpha_{i n}} h_{J}\left(k \omega_{L}+\frac{1}{2} \omega_{0}\right)\right) .
\end{aligned}
$$

The dc current components for the quasiparticle term exist only when $k=k^{\prime}$, whereas dc current components are found for the Josephson term when $\omega_{0}=n \omega_{L}$, where $n$ is an in- teger. The dc current resulting from the infuence of the microwave drive voltage, first derived by Werthamer, ${ }^{2}$ is

$$
\begin{aligned}
I_{\mathrm{dc}}\left(V_{0}\right)= & \sum_{k}^{\infty} J_{-\infty}^{2} J_{k}^{2}(\alpha) I_{4 \mathrm{p}^{2}}\left(k \omega_{L}+\frac{1}{2} \omega_{0}\right) \\
& +\sum_{n=0}^{\infty} \mid \sum_{k=1}^{\infty} J_{k}(\alpha) J_{n-k}(\alpha) I_{j} \\
& \times\left[\left(k-\frac{1}{2} n\right) \omega_{l}\right] \mid \delta\left(\omega_{0} \pm n \omega_{L}\right)
\end{aligned}
$$

The first term of this equation is obtained by substituting $k=k^{\prime}$ into the first term of Eq. (29) and taking the imaginary part of the resulting expression. The second term of this equation follows from the second tem of Eq. (29) by varying the initial phase $\alpha_{0}$ and restricting $n$ to positive integers. The maximum value of the dc current from the Josephson effect for the nth step is that given by the second term of $\mathrm{Eq}$. (30). Thus $\mathrm{Eq}$. (30) is the fundamental equation we need in order to study the zero-crossing step behavior at high frequencies. We can understand the first term of this equation better by noting that at the gap voltage $2 \mathrm{~A} / \epsilon$ the current increases dramatically for the quasiparticle portion of the $T$ $V$ curve of the junction $I_{4 p^{2}}$. Under the influence of a microwave field, the dc current increases rapidly at voltage intervals of $k$ h $w \omega_{L} / e$ from the gap voltage where the amplitudes of these terms are modulated by Bessel functions. Such current jumps are known as photon-assisted tunneling steps. ${ }^{13,14}$ Note that the microwave power affects the entire quasiparticle $I-V$ curve because at any bias voltage $V_{0}$ the current is comprised of contributions from other points on the $Z-V$ curve separated by voltages $V_{0}+k \hbar \omega_{L} / \ell$. The pair currents behave quite differently in that they modify the $l-V$ curve only at discrete voltages $n n \omega_{L} / 2 e$ with current-step halfwidth given by the second term of $\mathrm{Eq}$. (30).

The experimental consequences of Eq. (30) were explored initially by Hamilton ${ }^{7}$ who showed the expected effect of the peak in the supercurrent response on low-voltage $(n \sim 3)$ Josephson steps in tunnel junctions driven to large values of $\alpha(\alpha \geqslant 12)$ at low frequencies, $f_{L} \sim 25 \mathrm{GHz}$. The slow roll-off in $I_{J 1}$ at high frequencies was observed initially by McDonald et al. ${ }^{\circ}$ and in more detail by Weitz and coworkers, ${ }^{9}$ both using $\mathrm{Nb}$ point contacts. Later when it became possible to fabricate high-quality submicrometer-area tunnel junctions, Danchi and co-workers ${ }^{10}$ and Habbal and co-workers ${ }^{11}$ were able to demonstrate the efiects of $\mathbb{E q}$. (30) at low values of $\alpha(\alpha \sim 1)$ and at high frequencies $\left(f_{z}=604 \mathrm{GHz}\right)$. All of these experimental results demonstrate convincingiy that the Werthamer theory is the appropriate theory for the analysis of the behavior of tunnel junctions at high frequency, and that such analyses are on very firm ground. Equation (30) can now be used in a detailed analysis of the voltage standard which is independent of any low-frequency assumptions.

\section{Zero-crossing steps at high frequencies}

The condition for zero-crossing steps at high frequencies is the same as for the low-frequency RS model, namely, that the half-width of the Josephson step is greater than the 
quasiparticle current at the dc voltage of the Josephson step. Thus from Eq. (30) that condition is

$$
I_{J}^{n}>I_{\mathrm{qp}},
$$

where we introduce the notation

$I_{j}^{n}=\left|\sum_{k-\infty}^{\infty} J_{k}(\alpha) J_{n-k}(\alpha) I_{J !}\left[\left(k-\frac{1}{2} n\right) \omega_{L}\right]\right|$

and

$$
I_{\mathrm{qp}}=\sum_{k=-\infty}^{\infty} J_{k}^{2}(\alpha) I_{\mathrm{qp} 2}\left(k \omega_{L}+\frac{1}{2} \omega_{0}\right)
$$

In general, condition (31) can only be solved numerically. To make the problem analytically tractabie, we will make the assumption that the microwave drive voltage $\alpha \equiv e V_{L} / \hbar \omega_{L} \leqslant 1$; we then expand the Bessel functions for this limit. We also choose a frequency that is at or near the Riedel peak, i.e., $\omega_{L}=4 \Delta / \hbar$, because the presence of the Riedel peak gives the step amplitudes a logarithmic divergence at the gap frequency $4 \Delta / \hbar$. This region of frequency space has been unexplored in previous work. Before proceeding further with this analysis we find it useful to make a few notational improvements. We scale the real part of the Josephson response function $I_{J 1}$ to the critical current by defining $\tilde{I}_{J 1} \equiv I_{J 1} / I_{C}$. We also convert from frequency to voltage for the imaginary part of the quasiparticle response function $I_{\mathrm{qp} 2}$ by defining $I_{0}\left(V_{0}\right) \equiv I_{\mathrm{cp} 2}\left(\omega_{0} / 2\right)$ and using $\omega_{0}=2 e V_{0} / \hbar$. Under these conditions the most important term in Eq. (32a) is

$$
I_{J}^{\prime}=2 I_{C} J_{0}(\alpha) J_{1}(\alpha) \tilde{I}_{j 1}\left(\hbar \omega_{L} / 2 e\right) \text {. }
$$

To third order in $\alpha, \mathrm{Eq}$. (33) yields

$$
l_{J}^{1}=I_{C} \alpha\left(1-3 \alpha^{2} / 8\right) \tilde{I}_{J 1}\left(h \omega_{L} / 2 e\right) \text {. }
$$

In a similar way one can write Eq. (32b) as

$$
\begin{aligned}
I_{\mathrm{qp}}= & J_{0}^{2}(\alpha) I_{0}\left(V_{0}\right)+J_{1}^{2}(\alpha)\left[I_{0}\left(V_{0}+\hbar \omega_{L} / e\right)\right. \\
& \left.+I_{0}\left(V_{0}-\hbar \omega_{L} / e\right)\right] .
\end{aligned}
$$

Expanding ( 35 ) to lowest order in $\alpha$, we obtain

$$
\begin{aligned}
I_{\mathrm{qp}}= & \left(1-\alpha^{2} / 2\right) I_{0}\left(V_{0}\right)+\left(\alpha^{2} / 4\right)\left[I_{0}\left(V_{0}-\hbar \omega_{L} / e\right)\right. \\
& \left.+I_{0}\left(V_{0}-\hbar \omega_{L} / e\right)\right] .
\end{aligned}
$$

Inserting $\omega_{L}=4 \Delta / \hbar$ and $\nabla_{0}=2 \Delta / e$ into Eqs. (34) and (36) we obtain

$$
\begin{aligned}
& I_{J}^{1}=I_{C} \alpha\left(1-3 \alpha^{2} / 8\right) \tilde{I}_{J 1}(2 \Delta / e), \\
& I_{4 \mathrm{P}}=\left(1-3 \alpha^{2} / 4\right) I_{0}(2 \Delta / e)+\left(\alpha^{2} / 4\right) I_{0}(6 \Delta / e) .
\end{aligned}
$$

Ideally $\tilde{I}_{J_{1}}\left(V_{0}\right)$ is infinite at $V_{0}=2 \Delta / e$. The quasiparticle current $I_{\mathrm{qp}}$ can be at most

$$
\left[\left(1-3 \alpha^{2} / 4\right)+\left(3 \alpha^{2} / \pi\right)\right]\left(\pi \Delta / 2 e R_{N}\right) .
$$

where we have used the vaiue of the current just above the gap voltage for an ideal junction, given in $\mathrm{Eq}$. (22), which is $I_{0}(2 \Delta / e)=\pi \Delta / 2 e R_{N}$. At voltages significantly above the gap voltage the resistance approaches the normal-state resistance, so we approximate $I_{0}(6 \Delta / e)$ by $6 \Delta / e R_{N}$. Thus from inequality (3I) and Eqs. (37) we conclude that zero-crossing steps can exist up to and possibly beyond the frequency $4 \Delta / \hbar$. Ultimately the maximum frequency in which zerocrossing steps occur depends on how severely the Riedel peak is rounded for real (nonideal) junctions. For example, in real junctions, quasiparticle lifetime effects, gap anisotropy, and sample inhomogeneities round the Riedel peak as well as the quasiparticle $I-V$ curve ${ }^{19,20}$ After deriving a numerical estimate of the value of $\tilde{I}_{J 1}$ necessary for zero-crossing steps for ideal junctions, we analyze how this condition is modified for a nonideal junction.

From inequality (31) and Eqs. (37) we conclude that for small $\alpha$, the amplitude of the supercurrent at the gap frequency must be at least as great as

$\tilde{l}_{J 1} \frac{2 \Delta}{\epsilon} \geqslant\left[1+\left(\frac{3}{\pi}-\frac{3}{4}\right) \alpha^{2}\right] \alpha^{-1}\left(1-\frac{3 \alpha^{2}}{8}\right)^{-1}$.

A simple numerical estimate of this amplitude can be found from (38) assuming $\alpha \sim 1$. We observe that for a junction with ideal quasiparticle $l-V$ characteristics, the value of the pair response function $\tilde{I}_{J 1}$ at the gap frequency must fulfill the inequality, $\tilde{I}_{J !}(2 \Delta / e) \geqslant 1.93$. By numerically calculating the minimum of the right-hand side of (38) we find that $\tilde{I}_{J 1}(2 \Delta / e) \geqslant 1.85$ for $\alpha=0.85$. Of course, this inequality is easily fulfilled in this case since we have assumed a junction with ideal characteristics; $\tilde{I}_{j 1}$ by Eq. (23) is infinite at the gap voltage.

For real junctions, condition (38) can be used to determine a limit for the amount of rounding of the Riedel peak, when the rounding is described by a simple analytically derived parameter. Commonly used models of Riedel peak rounding invoke Lorentzian or Gaussian rounding of the logarithmic singularities $T_{J}(\omega)$ in Eq. (23). We use a form of rounding parameter similar to that of Zorin, Likharev, and Turovets. ${ }^{20} \mathrm{In} \mathrm{Eq.} \mathrm{(23)} \mathrm{we} \mathrm{make} \mathrm{the} \mathrm{following} \mathrm{substitutions:}$

$$
\begin{aligned}
& x=\frac{e V}{2 \Delta} \rightarrow 1-\left[\left(1-\frac{e V}{2 \Delta}\right)^{2}+\left(\delta \frac{e V}{2 \Delta}\right)^{2}\right]^{1 / 2}, x<1, \\
& \frac{1}{x}=\frac{2 \Delta}{e V} \rightarrow 1-\left[\left(1-\frac{2 \Delta}{e V}\right)^{2}+\left(\delta \frac{2 \Delta}{e V}\right)^{2}\right]^{1 / 2}, x>1 .
\end{aligned}
$$

The resulting line shape is approximately Lorentzian in the vicinity of the gap voltage. The parameter $\delta$ controls the height of the Riedel peak as well as its half-width. At $x=1$, the real part of the pair response function becomes

$$
\operatorname{Re} I_{s}(\omega)=\tilde{I}_{J 1}=(2 / \pi) K(1-\delta) \text {. }
$$

For small $\delta_{\text {; }}$

$$
K(1-\delta) \approx(1 / 2) \ln (8 / \delta)
$$

A variety of effects which contribute to the rounding of the Rieciel peak also smooth the quasiparticle $I-V$ curve. Such smoothing occurs usually in two ways. One is that the abrupt jump in the quasiparticle current at the gap given in Eq. (22) for $\operatorname{Im}=I_{\mathrm{qp}}(\omega)=I_{0}\left(V_{0}\right)$ is smeared out symmetrically to a finite width of $\sim 2 \delta$. The other is that quasiparticle leakage currents are found at voltages below the gap voltage. Impurities in the superconducting electrodes or barrier oxides produce quasiparticle subgap leakage currents which can be described by a resistance typically 5-10 times the normal state resistance. Both kinds of smoothing effects may change the value of the pair response function $\tilde{I}_{J}$ needed to fulfill the condition (31), which for the ideal junction had to be greater than 1.93. Equations (37) describe the Josephson step amplitude as well as the amplitude of the 
photon-assisted quasiparticle tunneling steps. We observe that the rounding of the quasiparticle $I-V$ curve affects the zero-crossing condition primarily from the value of the quasiparticle current at the gap voltage. At this high a frequency, subgap leakage currents do not alter the value of the pair response function $\tilde{l}_{J 1}$ needed for such steps; however, they may be more important particularly at lower frequencies. The rounding mechanisms discussed above give a total jump in current from just below to just above the gap voltage of magnitude close to that used previously, i.e, $\pi \Delta / 2 e R_{N}$. At the gap voltage itself, the current is about one-half of the total jump because the smoothing is symmetrical, so we use $I_{0}(2 \Delta / e)=(l / 2) \pi \Delta / 2 e R_{N}$.

Using this other estimate of $I_{0}(2 \Delta / e)$, condition (31) and Eq̨s. (37) yield, after substitution for $\alpha \sim 1$,

$$
\tilde{I}_{i 1} \frac{2 \Delta}{e} \geqslant \frac{4}{5}\left[1+\left(\frac{6}{\pi}-\frac{3}{4}\right)\right]=1.73 \text {. }
$$

A better estimate for the minimum value of $\tilde{l}_{s}$ can be found numerically, yielding a lower value for $\tilde{I}_{J 1}$ than by using $\alpha \sim 1$. This value is $\tilde{I}_{J 1} \geqslant 1.36$ for $\alpha=0.63$. We see that the value of the pair response function needed to obtain zerocrossing steps is somewhat lower with the rounded quasiparticle $\bar{l}-V$ curve than we obtained with the ideal quasiparticle $I-V$ curve. Substivuting for the rounded value of $\tilde{I}_{J 1}(2 \Delta / e)$ allows us to derive a limit

$$
(1 / \pi) \ln (8 / \delta) \geqslant 1.73
$$

for the value of the Riedel peak rounding parameter allowed for zero-crossing steps to occur. Condition (40) yields an upper limit of $\delta \leqslant 0.035$ for $\tilde{I}_{j 1}=1.73$. A more precise estimate for $\tilde{I}_{I 1}$, based on minimizing $\tilde{I}_{J 1}$ with respect to $\alpha$ as above, is $\tilde{I}_{J 1} \geqslant 1.36$. Hence, condition (40) yields the inequality $\delta \leqslant 0.11$, when this numerically determined value for $\tilde{I}_{J 1}$ is substituted on the right-hand side of $(40)$. Values of $\delta$ that are below this limit are found in real junctions. For example, Zorin and co-workers ${ }^{20}$ have used $\delta=0.050$ to realistically simulate experimental $I-V$ curves of small-area, high-current density junctions. Thus zero-crossing steps should be observable in real high-current density junctions. In a later section we show that zero-crossing steps can be found for $Y-V$ curves that are significantly more rounded than typical high-quality junction $I-V$ curves measured experimentally. We now analyze the effects of an ac curreni source on the behavior of the Josephson steps using a formulation of the problem that is correct at high frequencies.

\section{E. ac current source effects at high requencies}

We investigate the cffects of an ac current source with a method essentially similar to that used by $\mathbb{K}$ ant $z$ to derive Eqs. (13)-(15) for the Bessel function step amplitudes. In order to have the greatest similarity between our discussion of ac current source effects for the RSJ model and for the Werthamer theory, we recast Werthamer's equation for the current through the junction [our Eq. (20)] in the timedomain form discussed by Harris ${ }^{16}$ and Tucker and Feldman..$^{22}$ We use Tucker and Feldman's notation for the quasiparticle response of the junction. In the time domain, Eq. (20) for the current through a Josephson junction becomes

$$
I_{I J}(t)=V(t) / R_{N}+I_{\mathrm{qp}}^{d}(t)+\tilde{I}_{J}(t),
$$

where we define

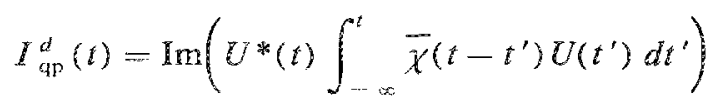

and

$$
\tilde{I}_{s}(t)=\frac{1}{2 \pi} \operatorname{Im}\left(U(t) \int_{-\infty}^{t} I_{J}\left(t-t^{\prime}\right) U\left(t^{\prime}\right) d t^{\prime}\right) .
$$

The functional $U(t)$ describes the time evolution of the junction and is given by

$$
\begin{aligned}
U(t) & =\exp \left(\frac{-i e}{\hbar} \int_{\infty}^{t} V\left(t^{\prime}\right) d t^{\prime}+\frac{i \phi_{0}}{2}\right) \\
& =\exp \left[i \phi_{s}(t)\right],
\end{aligned}
$$

where $\phi_{13}$ is a constant initial phase. Here $V(t)$ includes the de voltage contribution $V_{0}$ in addition to the time-varying voltage $V(t)$. The functional $U(t)$ depends on the entire past history of the junction. The kernels in Eqs. (42) and (43), $\bar{X}(t)$ and $h_{j}(t)$, are the causal response functions which deseribe the intrinsic time delays in the Josephson junction.

The function $\bar{X}(t)$ can be written in terms of the de $I-Y$ curve of the junction, i.e., $I_{4 p 2}$ as

$$
\begin{aligned}
\bar{X}(t)= & \frac{1}{\pi}(1+\operatorname{sgn} t) \int_{0}^{\infty}\left[\mathcal{I}_{\mathrm{qp} 2}(\omega)-G_{N} \hbar \omega / e\right] \\
& \times \sin (\omega t) d \omega,
\end{aligned}
$$

where $\operatorname{sgn} t=1$ for $t>0$ and $\operatorname{sgn} t=-1$ for $t<0$. In this way we explicitly show that $\bar{\chi}(t)=0$ for $t<0$. The quantity $\bar{X}(t)$ can be written in terms of only the imaginary part of the complex response function because the response functions satisfy the Kramers-Kronig transforms. Both the real and imaginary parts of the response function contribute equally to $\bar{\chi}(t)$, which is a real function. In a similar way, $\tilde{l}_{3}(t)$, the response function for the pair currents, can be written in terms of the cosine transform of the real part of the complexvalued response function for $Z_{J}(\omega)$ [recall that $\left.I_{J 1}=\operatorname{Re} I_{j}(\omega)\right]$, given in Eq. (23):

$$
I_{s}(t)=2(1+\operatorname{sgn} t) \int_{0}^{\infty} I_{j l}(\omega) \cos (\omega t) d \omega
$$

The equation we wish to solve is the analog of Eq. (3), the RSI model equation for a current-biased junction:

$C \frac{d V}{d t}+\frac{V}{R_{X}}+I_{\mathrm{qp}}^{d}(t)+\tilde{I}_{3}(t)=I_{0}+I_{1} \cos \omega_{L} t$.

To keep the closest possible analogy to the RSI model derivation discussed in Sec. II we separate the instantaneous response of the quasiparticies $V(t) / R_{N}$ from the delayed response $I_{\mathrm{qp}}^{d}(t)$. It is the delayed response which causes the photon-assisted tunneling steps which are not included in the RSJ model. Additional reactances, which are familiar in quantum mixer theory, also come from this term. ${ }^{22}$ We assume now that the current through the Josephson element $\tilde{I}_{J}(b)$ is small compared to the currents through the other elements of the junction. We assume also that the voltage across the junction is sinusoidal, but with unknown de and ac amplitudes $V_{0}, V_{l}$, and phase $\phi_{d}$ : 


$$
V(t)=V_{0}+V_{L} \cos \left(\omega_{L} t+\phi_{d}\right) .
$$

We solve for $V_{0}, V_{k}$, and $\phi_{d}$ given $I_{0}$ and $I_{1}$, as well as the other junction parameters. Given this $V(t)$, then $U(t)$ becomes

$U(t)=e^{-i e V_{0} l / \hbar} e^{+i \phi_{k} / 2} \sum_{k=-\infty}^{\infty} J_{k}(\alpha) e^{-i k\left\lfloor\omega_{1} t+\phi_{t}\right)}$,

where $J_{k}(\alpha)$ are Bessel functions of order $k$. When this $U(t)$ is substituted into Eq. (42) for the delayed part of the quasiparticle response, $I_{\mathrm{qp}}^{d}(t)$, then after some manipulation Eq. (47) reduces to

$$
\begin{aligned}
& \left(G_{N} V_{L}+G_{\omega}\right) \cos \left(\omega_{L} t+\phi_{d}\right)+\left(-\omega_{L} C V_{L}+B_{\omega}\right) \\
& \quad \times \sin \left(\omega_{L} t+\phi_{d}\right)=I_{1} \cos \omega_{L} t
\end{aligned}
$$

where $G_{N} \equiv R_{N}{ }^{1}, J_{k} \equiv J_{k}(\alpha)$, and $G_{\omega}$ and $B_{\omega}$ are given by

$G_{\omega}=\sum_{k=-\infty}^{\infty} J_{k}\left(J_{k+1}+J_{k-1}\right) I_{\mathrm{qp}}^{o}\left(V_{0}+k \hbar \omega_{L} / e\right),(50 \mathrm{a})$

$B_{c i}=\sum_{k-\infty}^{\infty} J_{k}\left(J_{k+1}-J_{k-1}\right) I_{\mathrm{KK}}\left(V_{0}+k \hbar \omega_{L} / e\right),(50 b)$

and where $I_{\mathrm{KK}}$ is the Kramers-Kronig transform of $I_{\mathrm{qp}}^{0}(V)$ defined by $I_{\mathrm{qp}}^{0}(V) \equiv I_{\mathrm{qp} 2}(V)-G_{N} V \cdot I_{\mathrm{qp}}^{0}$ is defined in this way to make the integral for $\bar{\chi}(t)$ a proper (finite) Fourier transform. If the divergent part of the quasiparticle response function were not subtracted, the Fourier transform would be ill defined. The de current through the junction must satisfy the equation

$$
I_{0}=\sum_{k=-\infty}^{\infty} J_{k}^{2}(\alpha) I_{\mathrm{4p} 2}\left(V_{0}+k h \omega_{i} / e\right)
$$

This equation can be obtained from the dc component of the quasiparticle current when Eq. (48) is substituted into Eq. (42) and the de components of Eq. (47) are collected together.

By squaring both sides of Eq. (49) and time averaging we can derive a relation for $V_{l}$ and hence $\alpha$, given $I_{1}$,

$$
\left(G_{N} V_{L}+G_{\omega}\right)^{2}+\left(-\omega_{L} C V_{L}+B_{\omega}\right)^{2}=I_{1}^{2} .
$$

Equation (51) is familiar as the large-signal problem in quantum mixer theory in which one determines the operating point of the mixer given a large local oscillator drive. ${ }^{22}$ In general, Eq. (51) can be solved self-consistently by calculating $B_{\omega}$ and $G_{\omega}$ given an initial value for $V_{L}$ and the known values $I_{1}, C, G_{N}$, and $\omega_{L}$. We can also make approximate solutions to (51) for low microwave power. In this limit we retain only the $k=0,1,-1$ terms of ( $50 a)$ and $(50 b)$ in adaition to expanding the Bessel functions only to low order in $\alpha$.

The phase angle between the voltage and the current $\phi_{a t}$ can be found from Eq. (49) and is given by

$$
\tan \phi_{d}=\left(\frac{\omega_{L} V_{L} C-B_{\omega}}{G_{N} V_{L}+G_{\omega}}\right)
$$

In the low-power limit (first order in $\alpha$ ), Eqs. (50) for $G_{w}$ and $B_{c o}$ can be written

$$
G_{\omega}=V_{L} G_{1} \text {, }
$$

where

$$
\begin{aligned}
G_{1}= & e / 2 \hbar \omega_{L}\left[I_{0}\left(V_{0}+\hbar \omega_{L} / e\right)-I_{0}\left(V_{0}-\hbar \omega_{L} / e\right)\right. \\
& \left.-2 G_{N} \hbar \omega / e\right]
\end{aligned}
$$

and

$$
B_{\omega}=V_{L} B_{1},
$$

where

$$
\begin{aligned}
B_{1}= & e / 2 \hbar \omega_{L}\left[-I_{\mathrm{KK}}\left(V_{0}+\hbar \omega_{L} / e\right)\right. \\
& \left.-I_{\mathrm{KK}}\left(V_{0}-\hbar \omega_{L} / e\right)+2 I_{\mathrm{KK}}\left(V_{0}\right)\right] .
\end{aligned}
$$

The relation between the driving current and an equivalent voltage source can be written in the low-power limit as

$\alpha=\frac{e V_{L}}{h \omega_{L}}=\frac{e I_{1}}{\hbar \omega_{L} \sqrt{\left(G_{N}+G_{1}\right)^{2}+\left(\omega_{L} C-B_{1}\right)^{2}}}$.

At low voltages and low frequencies, $V_{0} \ll 2 \Delta / e$ and $\hbar \omega_{R} \ll 2 \Delta$, the conductance $G_{1}$ becomes $G_{1}=\left(G_{0}-G_{N}\right)$, where $G_{0}=d I_{0}\left(V_{0}\right) / d V$, and $B_{1} \approx 0$. Hence (55) becomes

$$
\alpha=\frac{e V_{L}}{\hbar \omega_{L}}=\frac{e I_{1}}{\hbar \omega_{L} \sqrt{\left(G_{0}\right)^{2}+\left(\omega_{L} \bar{C}\right)^{2}}} .
$$

When Eq. (56) is converted to normalized units it is equivalent to Eq. (17) derived earlier for the relationship between the microwave drive current $I_{1}$ and the equivalent microwave drive voltage $\alpha$ in the context of the RSJ model. At high frequencies, the relationship between $I_{1}$ and $\alpha$ is somewhat different. For example, suppose $\omega_{L}=4 \Delta / \hbar$ and $V_{0}=2 \Delta / e$; in this case $G_{1}$ and $B_{1}$ become

$$
\begin{aligned}
& G_{1} \sim\left(G_{N} / 8\right)[(\pi / 2)-2], \\
& B_{1} \sim(e / 8 \Delta) I_{\mathrm{KK}}(2 \Delta / e) .
\end{aligned}
$$

We conclude that in this context $\left|G_{1}\right| \leqslant G_{N} . B_{1}$ can be evaluated for a rounded quasiparticle $I-V$ curve. In the same way in which the Riedel peak is rounded, the logarithmic divergence of $I_{\mathrm{KK}}$ at $2 \Delta / e$ is rounded as well. With the rounding parameter $\delta$ described earlier, we obtain the following approximation for $I_{\mathrm{KK}}(2 \Delta / e)$ :

$$
I_{\mathrm{KK}}\left(\frac{2 \Delta}{e}\right) \sim \frac{\Delta}{e R_{N}}\left[\frac{1}{2} \ln \left(\frac{8}{\delta}\right)-2\right] .
$$

For $\delta \sim 0.1$, the largest $\delta$ or the most rounded Riedel peak that one would expect, we find $Y_{\mathrm{KK}}(2 \Delta / e) \sim 0.19\left(\Delta / e R_{N}\right)$. For $\delta \sim 0.001$, the situation where there is very little rounding, we obtain $I_{\mathrm{KK}}(2 \Delta / e) \sim 2.5\left(\Delta / e R_{N}\right)$. We see that the divergence at $2 \Delta / e$ does not represent a major problem, because if the capacitive reactance is small compared to the reactance associated with the Josephson inductance (a necessary condition for Bessel function step amplitudes) then we have fulfilled the condition needed at high frequencies to minimize the reactance of the quasiparticles relative to the capacitive reactance. Another way to look at it is directly from Eq. (55). Consider first that

$$
B_{1}=\frac{e I_{C}}{\pi h \omega_{L}}\left[\frac{1}{2} \ln \left(\frac{8}{\delta}\right)-2\right]
$$

for frequencies near the gap frequency. Therefore, in order to satisfy $\omega_{L} C \gg B_{1}$, the condition 


$$
\Omega^{2} \beta \gg \frac{1}{2 \pi}\left[\frac{1}{2} \ln \left(\frac{8}{\delta}\right)-2\right]
$$

must be satisfied or equivalently the quasiparticle $I-V$ curve must have a minimum rounding given by

$$
\delta \geqslant 8 \exp -2(2 \pi+2) \sim 5.1 \times 10^{-7} .
$$

This condition is easily fulfilled for most practical junctions. Equation (55) yields

$$
\alpha=\frac{e I_{1}}{n \omega_{L} \sqrt{\left(G_{N}\right)^{2}+\left(\omega_{L} C\right)^{2}}},
$$

the high-frequency relation between driving current and equivalent ac voltage. Equation (58) is completely analogous to the low-frequency equation (56); the only difference is that the dissipative element of the junction at high frequencies is essentially the normal-state resistance rather than the low-voltage dynamic resistance.

\section{F. Discussion}

It is useful to compare these results with other work performed at high frequencies. Danchi et $a l^{23}$ studied the behavior of $\mathrm{Sn}-\mathrm{SnO}-\mathrm{Pb}$ tunnel junctions irradiated at 604 GKz by a far-infrared molecular laser. They found remark. able agreement between their experimental $I-V$ curves and simulated $Y-V$ curves based on a current-biased RSI model with noise and with photon-assisted quasiparticle tumneling steps. These steps were put in by adding to the RSI model current at a particular voltage the current contribution from photon-assisted unneling. Here the photon-assisted tunneling contribution was that calculated for an rf voltage bias and a dc voltage bias. The quality of the agreement between the simulation and data was surprising considering that their procedure was somewhat ad hoc. They argued that the junction was effectively voltage biased because of the large value of the $\omega_{L} R_{N} C$ product for their junctions at the $604 \mathrm{GHz}$ laser frequency. The analytic calculation discussed in Secs. II $\mathrm{D}$ and $\mathrm{II}$ E rigorously shows that the procedure they used was correct. Another problem with their analysis was in determining the correct impedance for the calculation of $\alpha$ from the if drive current. In the formulation of the problem we have used here, this impedance comes ont quite naturally.

\section{PRACTICAL CONSIDERATIONS}

For a voltage standarc at high frequencies to actually work in practice, several conditions must be fulfilled. The most significant are that superconducting tunnel junctions of sufficient quality and reliability must be fabricated, that the standard must maintain a stable voltage, and that suffcient microwave drive power should be available at the desired frequency. Stability on the zero-crossing step is perhaps the most significant factor that affects the whoie design, as has been discussed thoroughly by Kautz, Hamilton, and Lloyd. ${ }^{24}$ We apply some of the methods of their analysis to the situation of interest here: that of a junction being driven at frequencies near the gap frequency. These authors have discussed the design of the individual junctions that make up the standard in terms of junction current density $J_{c}$, length $l$, and width $w$. We briefly review these requirements for com- pleteness of this discussion. To obtain Bessel function step amplitudes, the junction must be driven at a frequency above the plasma frequency [condition (11)]. This condition can be interpreted as a requirement on the current density of the junction, ${ }^{24}$ because the ratio of the critical current to capacitance in the expression for the plasma frequency is independent of the junction area. Otherwise if the driving frequency is close to the plasma frequency then chaos has been observed both in simulation at low frequencies ${ }^{25}$ and in experiments at submilimeter wavelengths. ${ }^{26} \mathrm{Kautz}$ and coworkers ${ }^{24}$ describe a normalized parameter which is the ratio of the critical current density $J_{c}$ to the product of the square of the drive frequency $\omega_{L}^{2}$ and the specific capacitance $C_{s} \equiv C / w l$. This parameter which we call $\gamma$ is given by

$$
\gamma \equiv\left(\omega_{p} / \omega_{L}\right)^{2}=2 e J_{c} / \hbar \omega_{L}^{2} C_{s} .
$$

Monte Carlo simulations have been performed which indicate that a good design choice for the value of $\gamma$ is $\gamma \sim 0.1$, aithough those studies indicate $\gamma$ can take on values from 0.1 to 0.3 because there is a broad minimum in the mean time to jump from the Josephson step in these regions. ${ }^{24}$ A separate set of time-domain simulations, performed with values of $\gamma$ ranging from 0.1 to 1.0 , indicate that at high frequencies the locking range is most ideal (close to the Werthamer theory) when $\gamma \leqslant 0.3$, so it appears that the choice of $\gamma=0.1$ is a sensible one for high frequencies in the absence of further Monte Carlo simulations. For junctions made from $\mathrm{Nb}$ base electrodes with an $\mathrm{Nb}_{2} \mathrm{O}_{5}$ barrier, the specific capacitance is roughly $C_{s} \approx 14 \mu \mathrm{F} \mathrm{cm}^{-2} .{ }^{27}$ Using this value of specific capacitance and a driving frequency $\omega_{L}=7.9 \times 10^{12} \mathrm{~s}^{-1}$, we find that the critical current density should be $J_{c} \approx 2.9 \times 10^{4}$ A $\mathrm{cm}^{-2}$.

For a bias point at the center of the nth Josephson step, Fokker-Planck methods have been used to find the mean time between phase slips $\tau$, which occur when the junction loses lock to the radiation field due to the thermal activation processes ${ }^{25}$ (essentially the Johnson noise in the resistive element $R$ ). This $t$ is also approximately the mean time for the junction to jump between Josephson steps and is given by

$$
\tau=\tau_{0} e^{E / k r},
$$

where $k T \ll E$, and where $E_{\text {s }}$ the activation energy, and $\tau_{0}$, the attempt time, are given by

$$
E=\hbar \Delta I_{n} / 2 e
$$

and

$$
\tau_{0}=\left(\pi / \omega_{p}\right) \sqrt{2 I_{C} / \Delta I_{n}} .
$$

In this model $\Delta I_{n}$ indicates the full width of the Josephson steps. For the RSI model, $\Delta I_{n}=2 I_{C}\left|J_{n}(2 \alpha)\right|$, whereas in the Werthamer theory the full width is $2 I_{j}^{n}$, where $I_{j}^{n}$ is given by (32a). At high frequencies, the simple expressions (60)-(63) may not completely deseribe the behavior of the junction since shot noise may also significantly perturb the Josephson currents. ${ }^{23}$ However, for the purpose of this work, which is to explore roughiy the differences between the lowand high-frequency escape time $\tau$, this type of analysis is sufficient. A simple way to take into account the effects of shot noise is to define an effective temperature $T_{n}^{\text {eff }}$ for the $n$th step, such that the mean-square noise current from shot 
noise is equal to that from a resistor at that elevated temperature, i.e.,

$$
\left\langle i_{n}^{2}\right\rangle_{\text {shot }} \sim 2 e V / R_{N V} \equiv\left\langle i_{n}^{2}\right\rangle_{\text {therm }} \sim 4 k T T_{n}^{\text {eff }} / R_{N}
$$

Thus $T_{n}^{\mathrm{eff}}=e V_{n} / 2 k$, and since the voltage at the $n$th step is $V_{n}=n \hbar \omega_{L} / 2 e, T_{n}^{\text {eff }}=n\left(\hbar \omega_{L} / 4 k\right)$. This gives $T_{i}^{e f f} \approx 15 \mathbb{K}$ for the first step at $\omega_{L}=4 \Delta / \hbar$.

The activation energy $E$ at twice the gap frequency becomes

$$
E=\left(\frac{h I_{C}}{e}\right) \frac{5}{4 \pi} \ln \left(\frac{8}{\delta}\right)
$$

where we have used the full width of the first step from Eq. (34),

$$
\Delta I_{1}=(5 / 4 \pi) I_{C} \ln (8 / \delta)
$$

which is appropriate for $\alpha \sim 1$, near the maximum width of this step. The attempt time $\tau_{0}$ can also be evaluated and yields

$$
\left.\tau_{0}=\left(\pi / \sqrt{\gamma} \omega_{L}\right) \sqrt{[8 \pi / 5} \ln (8 / \delta)\right]
$$

Before evaluating Eqs. (60), (64), and (65) we turn briefiy to the optimum dimensional parameters of the junction. The stability of the phase-lock state is also affected by the length and width of the junction. The microwave currents in the usual geometry for a series voltage standard propagate along the length of the junction and produce a magnetic field in the junction dielectric, which causes a nonconstant junction phase that grows quadratically along the junction axis. ${ }^{24}$ This time-varying phase reduces the critical current to an effective current lower than the original critical current and hence reduces the couping energy. The optimum junction length has been found to be

$$
l=\sqrt{\left(6 J_{c} / \omega_{L} C_{s}\right.} \cdot \overline{\left.V_{n}\right)} \lambda_{j},
$$

where $\lambda_{J}$ is the Josephson penetration depth defined by $\lambda_{3} \equiv\left(\hbar / 2 e \mu_{0} d J_{c}\right)^{1 / 2}$ and where $d$ is the sum of the dielectric thicknesses and the penetration depths of each electrode. ${ }^{24}$ Using $J_{c}=2.9 \times 10^{4} \mathrm{~A} \mathrm{~cm}{ }^{-2}$ and $d=230 \mathrm{~mm}$, the optimum junction length is $l=0.77 \lambda$. The upper limit of the junction width $w$ is set by the presence of Fiske modes, which occur beginning at the frequency $\omega_{F}=(\pi / v) \sqrt{\mu_{0}} d \overline{C_{5}}$. The junction width must be small enough that the first Fiske mode occurs at a frequency higher than that of the driving frequency $\omega_{L}$, otherwise these modes can be excited, reducing the phase lock of the junction. The upper limit on the junction widh is thus

$$
w=\pi / \omega_{L} \sqrt{\mu_{0} d C_{s}} .
$$

Evaluating (67), one finds that $w_{\max } \sim 2.0 \mu \mathrm{m}$.

If one evaluates the critical current $I_{C}$ from Eqs. (59), (66), and (67), one inds (using $I_{C}=\left(u J_{c}\right.$ )

$$
I_{C}=\sqrt{6} \pi \hbar y / 2 \mu_{0} e d,
$$

which depends on materials oniy through the length $d$. Taking $d \approx 230 \mathrm{~nm}$ appropriate for $\mathrm{Nb} / \mathrm{Nb}_{2} \mathrm{O}_{5} / \mathrm{PbInAu}$ junctions and $\gamma \approx 0.1$, the optimum critical current should be $\sim 0.87 \mathrm{~mA}$. Using the ideal $I_{C} R_{N}$ product for a junction with $\Delta=1.3 \mathrm{meV}$ yields a normal state resistance $R_{N}$ of 2.4
$\Omega$. The coupling energy $E$ of $E q$. (14) can be evaluated yielding

$$
E=\left(5 \sqrt{6} \hbar^{2} \gamma / 8 \mu_{0} e^{2} d\right) \ln (8 / \delta)
$$

Using $\delta=0.05$, which is likely to be the largest $\delta$ one would use in practice, and using the above-mentioned values for $d$, and $\gamma$, we find that $E / k=8.4 \times 10^{4} \mathrm{~K}$. Evaluating (65) for $\tau_{0}$ we find that $\tau_{0}=1.25 \times 10^{-12} \mathrm{~s}$. If we assume that thermal noise is the only source of phase slips and if we use $T_{\text {bath }}$ $=4.2 \mathrm{~K}$, then $E / k T_{\text {bat }}=2.0 \times 10^{4}$, whereas if $T_{!}^{\mathrm{eff}}=15$ $\mathrm{K}$, assuming that shot noise is the major cause of phase slips, then $E / k T_{1}^{\mathrm{N} T}=5.6 \times 10^{3}$. Both of these values are somewhat above that of the voltage standards operated at 96 GLIz, where $E / k T_{\text {bath }}=3.6 \times 10^{3}$. It is important to point out that it would be useful to analyze the behavior of the junction more fully when it is phase locked at such high driving frequencies. Based on this analysis high frequencies apparently have a significant advantage in terms of stability when locked onto the step; however, other choices exist for some of the parameters. For example, one could choose the optimum critical current based on other design strategies. Also, one has found an optimum value of $\gamma$ at high frequencies, so the complete answer to the question of stability is not known at present. Further work will be necessary to sort out this important issue. It is clear that fabricating junctions according to these design criteria should not be too difficult since the junction area would be less than or approximately equal to $4 \mathrm{~km}^{2}$ and the current densities (in the $10^{4} \mathrm{~A} \mathrm{~cm}^{-2}$ range) are not too high.

Coupling the electromagnetic field to an array of these junctions is a serious issue and points out some of the tradeoffs involved in designing a voltage standard to operate at high frequencies. One approach that has worked successfully is the planar bow-tie antenna on a quartz substrate, which is itself attached to a quartz hyperhemisphere. Such coupling structures have been used in studies of SIS mixers at submillimeter wavelengths. ${ }^{28}$ Another possibility is the Vee antenna, which is a planar antenna sandwiched between quartz substrates. ${ }^{29}$ A stripline configuration such as that used for the $96 \mathrm{GHz}$ voltage standard could also be used in a high-frequency standard. The power requirements are not too severe. The required microwave current is given by $\Gamma_{1}=\omega_{2} C V_{n}$, which for the values of capacitance and step voltage for the example in this work is $I_{1}=8.6 \mathrm{~mA}{ }^{24}$ Therefore, the drive power level $P_{L}=(1 / 2) I_{1}^{2} Z_{0}$, where $Z_{0}$ is the impedance of the stripline which could be designed to be in the range from $\sim 10$ to $100 \Omega$. If we assume that the stripline impedance is $100 \Omega$, then $P_{l}=3.7 \mathrm{~mW}$, while if we assume the lower value of stripline impedance, which is closer to that used in practice, $P_{L}=0.37 \mathrm{~mW}$. Far-infrared molecular lasers can produce well over $10 \mathrm{~mW}$ at frequencies near $1 \mathrm{THz}$, so it should be possible to couple enough power into the array if we assume coupling efficiencies in the range from $4 \%$ to $40 \%$, which should be achievable.

We conclude this section by noting that there does not appear to be any practical reason why voltage standards could not be made to operate at frequencies as high as 1.2 THz. It is ciear that some of the issues discussed herein de- 
serve further attention before a practical standard can be developed.

\section{MUMERICAL CALCULATIONS}

\section{A. Numerical calculations with the voltage-bias frequency-domain theory}

\section{Gap irequency drige}

The analytical calculations based on Eq. (30) in Secs. $\Pi D$ and $I \mathbb{E}$ provide a clear set of criteria that a junction must satisfy in order to have zero-crossing steps at the gap voltage. Numerical calculations based on Eq. (30) for the behavior of the ac Josephson steps at high frequencies can be made for model tunnel junction quasiparticle $I-V$ curves and a rounded Riedel peak. The pair-response function $\tilde{I}_{J 1}$ can be rounded in a straightforward manner as described in Sec. II D. The quasiparticle $I-V$ curves on the other hand must be modelled in such a way as to include both kinds of "smoothing" effects noted in Sec. IN D, the smoothing of the discontinuity at the gap voltage, as well as leakage currents below the gap voltage which can be modelled by a finite subgap resistance $R_{L}$. By controlling the subgap resistance, the "width" of the quasiparticle current rise at the gap voltage, and the height of the Riedel peak we simulate the frequencydomain expressions for a real junction. Later, in the timedomain simulations discussed in the next section, a fast Fourier transform of these expressions is used to calculate response functions in the time comain.

In Fig. $I$ we display realistic $l \times V$ curves and pair-current response functions used in the numerical computations. The I-V Curves used here are simulated but realistically rounded by the method of Solner and Powell ${ }^{30}$ and were used in numerical calculations of SIS quantum mixers by Danchi and Sutton. ${ }^{31}$ The $I-V$ curves and the Riedel peak are rounded using the parameter $\delta$, discussed previously, which takes on the values of $0,0.005,0.02$, and 0.05 . The gap-sharpness parameters $e^{T^{\prime} / 2 \Delta}$ and $e^{\prime \prime} / 2 \Delta$ described by Sollner and Powell are set equal to $\delta$, the parameter describing the rounding of the Riedel peak. In this way we have a single parameter to describe the rounding of both the quasiparticle $T-W$ curve and the Riedel peak. We are still allowed the freedom to independently adjust the subgap leakage resistance in order to separate the two effects on the zero-crossing behavior of the junctions. The subgap leakage resistance varies also in these $K-V$ curves with values $R_{L}=\infty, 100 R_{N}$, $25 R_{N}$, and $10 R_{N}$.

A numerical calculation of the Josephson step width based on the voltage-biased junction theory of Sec. II $D$ is shown in Figs. 2(a)-2(d). In this figure we display the up per and lower ends of the Josephson steps (black lines) for increasing values of $\alpha$, the microwave drive level. The vertical scale is in dimensionless current units in which the current is scaled to the critical current. From these plots we observe that the locking range depends on the rounding of the Riedel singularity. As expected, the more the singularity is rounded the smaller the locking range. We observe a locking range between $\alpha \sim 0.24$ and 1.47 for $\delta=0.005$, whereas the locking range is reduced to between $\alpha \sim 0.40$ and 1.13 for $\delta=0.05$. Note that the full width of the Josephson step is
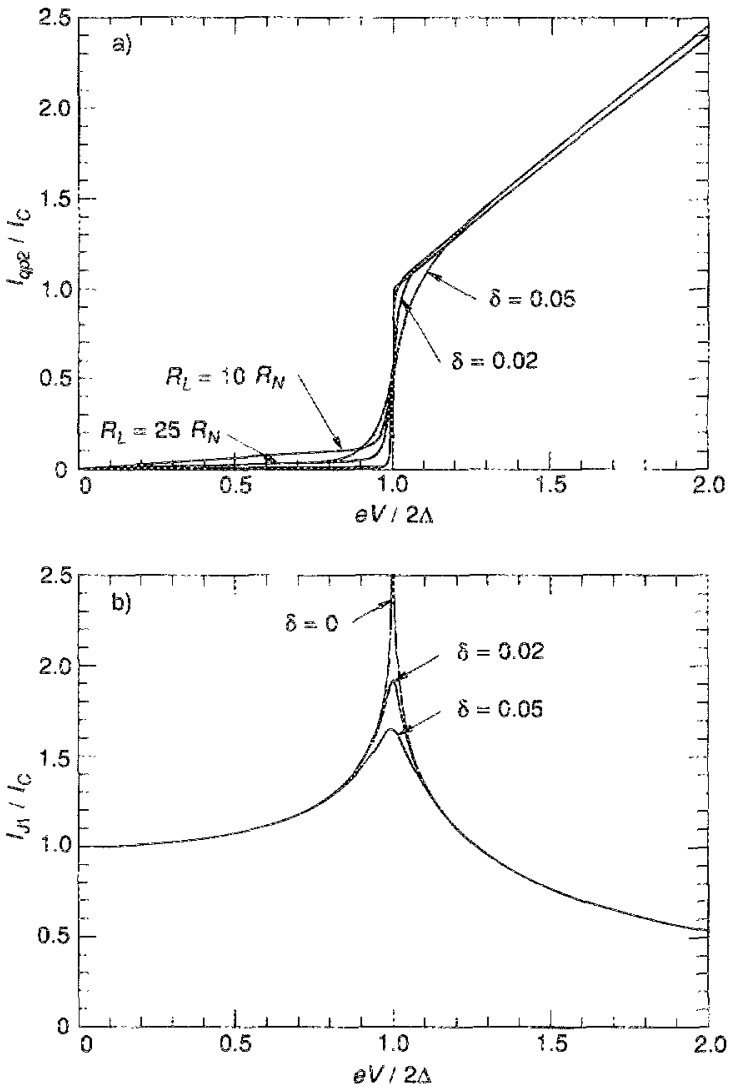

FIG. 1. (a) The imaginary part of the quasiparticle response function $I_{\mathrm{qp} 2}$ normalized to $I_{C}$ for the following values of phenomenological rounding parameters: (i) $\delta=0, R_{L}=\infty$, (ii) $\delta=0.005, R_{L}=100 R_{N}$, (iii) $\delta=0.020, R_{L}=25 R_{N}$, (iv) $\delta=0.020, R_{L}=10 R_{N}$, (v) $\delta=0.050$, $R_{L}=25 R_{N}$. (b) The real part of the pair response function $I_{J \ell}$ normalized to $\bar{C}_{C}$ for the same values of $\delta$ as in (a). Both (a) and (b) are computed for the voltage normalized to the gap voltage $e V / 2 \mathrm{~A}$.

about 2.3 times larger than the step width would be without the Riedel peak (from Bessel function RSJ expression) when $\delta=0.02$.

\section{Subhamonis dity}

Enhanced Josephson step widths for low-voltage steps can be found when the junction is driven by subhamonics of $4 \Delta / h$ as has been discussed by Hamilton. ${ }^{7}$ It may also be possible that the step width at the gap voltage is larger than the width expected from the RSI model. Analysis of Eq. (30) for the cases when $f_{L}=(1 / 2) 4 \Delta / h$ and $f_{L}$ $=(1 / 3) 4 / h$ shows that the step widths at the gap voltage indeed are expected to be larger than the RSI model step widths. For example, when $f_{L}=(1 / 2) 4 \Delta / h$, it is expected that the second Josephson step is enhanced by the Riedel peak but not the first or the third steps. Similarly, if $f_{I}$ $=(1 / 3) 4 \Delta / h$, then it is expected that the first and third steps are enhanced, but not the second step. These steps are enhanced by the Riedel peak because they fulfill the condition that $|2 k-n|=l$, where $l$ is an integer and where $f_{L}=l^{-1}(4 \Delta / h)$. This result is easily derived from (30) by noting the terms in the summation on $k$ in the argument of $\tilde{I}_{J 1}$ which equal the gap voltage $2 \Delta / e$. The symbol $n$ here is the step number and $k$ is the summation index in Eq. (30). 

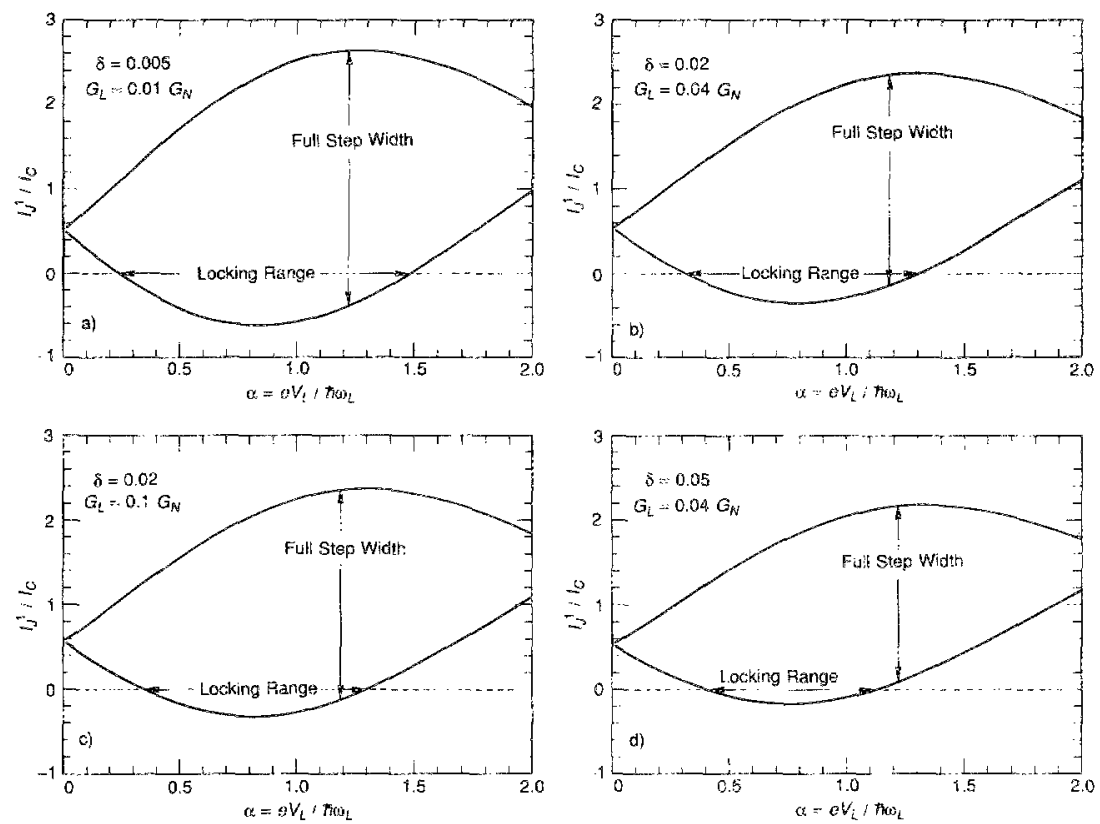

FIG. 2. Upper and lower ends of the Josephson steps calculated from the voltage-bias Werthamer theory are displayed in the black lines for increasing values of the microwave drive level $\alpha=e V_{L} / \hbar \omega_{L}$. The vertical scale is in dimensionless current units in which the step current is scaled to the critical current. (a) Here we have a very sharp but not ideal Riedel peak, with $\delta=0.005$, $R_{L}=100 R_{N}$. (b) A more realistic case with $\delta=0.02$ (corresponding to a peak height of about $1.9 I_{\mathrm{C}}$ ) and $R_{L}=25 R_{N}$. (c) Here $\delta=0.02, R_{I}=10 R_{N}$. (d) A highy rounded Riedel peak with $\delta=0.05$ and $R_{\varepsilon}=25 R_{N}$.
Thus if $l$ is an odd integer then only odd numbered steps should be enhanced, while if $l$ is an even integer then even numbered steps are enhanced.

This analysis is supported by numerical computations. Equation (30) can be evaluated for $\delta=0.02, G_{N}=0.02 \mathrm{~S}$, $G_{L} / G_{N}=0.04$, and $2 \mathrm{~A} / e=2.6 \times 10^{-3} \mathrm{~V}$, which is the same set of conditions as Fig. $2(b)$. We find the largest steps at the gap voltage when $n=1$ and $f_{L}=4 \Delta / h$. In this case the step width is maximized when $\alpha=1.1$ and the full step width is $2.5 I_{\mathrm{C}}$. This can be contrasted with the RSJ model width of $1.1 I_{c}$ at $\alpha=1.0$. When $l=2$, the step at the gap voitage is the $n=2$ step, which is maximized at $\alpha=1.5$ with full width $1.47 I_{C}$, while the RSJ model width is $0.97 I_{C}$. When $l=3$ the maximum value of the step at the gap voltage $(n=3)$ occurs at $\alpha=4.0$ with full width of $1.29 I_{C}$ as compared to an RSI width of $0.87 I_{C}$ for $\alpha=2$. I. Only when $l=n=1$ do we observe zero-crossing steps; the step widths are significantly increased for $l=3, n=3$ and $l=2, n=2$, but do not cross the zero-current axis. This means that in a series array voltage standard one would have to use some do bias current to utilize these steps. An interesting point concerns the first step $(n=1)$ when $f_{L}=(1 / 3) 4 \Delta / h$. We find that this step has a maximum width of $1.57 I_{C}$ for $\alpha=2.6$ as compared to a width of $1.16 I_{C}$ for $\alpha=1.0$ from the RSJ model. Thus it appears that driving the junctions with a subharmonic of twice the gap frequency has an advantage with regard to stability when locked on the step because the steps are so much larger than expected from the RSI mode!. We shall discuss stability questions in detail in Sec. III.

\section{B. Timedomain simulations}

\section{Numerical aporoach}

A set of FORTRAN programs has been written to simulate the behavior of a Josephson junction, described analytically by Eqs. (41)-(47). The algorithm for the programs is essentially as follows. Suppose the junction is located in an external circuit containing a capacitor and dc and ac current sources, and suppose that the voltage and phase are known at discrete intervals up to a time $t_{i}$. We wish to obtain the voltage and phase at the next time point. We can obtain the phase if we know the voltage, since from Eq. (44), $\dot{\phi}_{s}=-(e / \hbar) V(t)$. If we know the current through the junction and the contribution from the dc and ac current sources, we can compute the voltage from $l=C d V / d t$. The junction current is calculated from the convolution integrals [Eqs. (41)-(43)], since we know the values of $\phi$ for previous times. To ensure higher accuracy, it is desirable to include the phase at the current time point in this calculation; however, the phase is one of the quantities we are trying to determine. This problem is solved through an iterative process as follows. The voltage $V_{i}$ is defined to be the voltage at the ith time point $t_{i}, V_{i} \equiv V\left(t_{i}\right)$. The initial value of $V_{i}$ is based on a parabolic extrapolation from previous voltage points. The phase $\phi_{i}$ is calculated from the previous voltage and phases by

$$
\phi_{i}=\phi_{i-1}-\frac{1}{2} \frac{e}{\hbar}\left(V_{i}+V_{i \ldots 1}\right) \Delta t .
$$

Following this the convolution integrals are computed using response functions for the quasiparticles and for the pairs that had been calculated previously. This procedure yields $I_{J}$, the total junction current. The charge on the capacitor $Q_{i}$ due to the junction current is computed from

$$
Q_{i}=Q_{i-1}+\frac{1}{2}\left(I_{J_{i}}+I_{J_{i}}\right) \Delta t \text {. }
$$

Contributions to the total charge, $Q_{\text {tot }}$, from the dc and ac current sources are computed analytically from $I_{0} \Delta t$ and $\int I_{1} \sin (\omega t) d t$. The voltage is then $V_{i}=Q_{\text {iot }} / C$. Thus we have a new approximation to $V_{i}$ and the process is iterated. To speed the convergence of this process, an accelerated convergence technique adapted from the Aitken $\Delta^{2}$ process is used. ${ }^{32}$ Once the convergence criterion is satisfied, we cease iterating and record the voltage, phase, and current, and we then continue to the next time point. 
There are two methods used to compute the time-domain response functions depending on whether the junction is assumed to have ideal or realistic characteristics. For the ideal junction, the response functions can be computed from the Bessel function expressions of Harris ${ }^{17}$ given in his Eq. (2). For more realistic junctions, however, analytical expressions for the response functions do not exist, and we must compute Fourier transforms over the frequency-domain expressions. The time domain response functions are obtained by fast Fourier transformation of the rounded quasiparticle $I-V$ curves and pair response functions of Sec. IV A.

The simulations described below are begun at ime $t=0$, and it is assumed that the junction phase $\delta(t)=\phi_{0}=$ const, and $V(t)=0$ for $t<0$. The initial guess for $V(0)$ may be chosen to have any value; different choices may well lead to different steady-state solutions.

\section{Tests of the simulation}

A good test of a numerical simulation of a tunnel junction is that it produces realistic $I-V$ curves. By incrementing the bias current and taking the time average of the voltage waveform, the resulting $L-V$ curve should agree well with the predictions of the Werthamer theory and the RSJ model. We display in Fig. 3 an $I-V$ curve for an ideal junction, with $R_{N}$ $=50 \Omega, C=100 \mathrm{fF}$, and $\Delta / e=1.3 \mathrm{mV}$, corresponding to the energy gap of niobium. In this plot the initial voltage is set at zero for the lowest current point and the bias current is incremented using the time-averaged voltage from the previous point as the initial voltage. Note that the junction remains in the zero-voltage state until about $75 \%$ of the critical current, when it leaps up to the finite voltage state. Above the critical current, the junction displays an $Z$ - $V$ curve which agrees with the analytical expression from the Werthamer theory, Eqs. (22) and (23), to better than one part in one thousand. Starting at a current that is approximately twice the critical current, the bias current is decreased, once again using the time-averaged voltage from the previous poin as the initial voltage at each point. This $I-T$ curve displays a

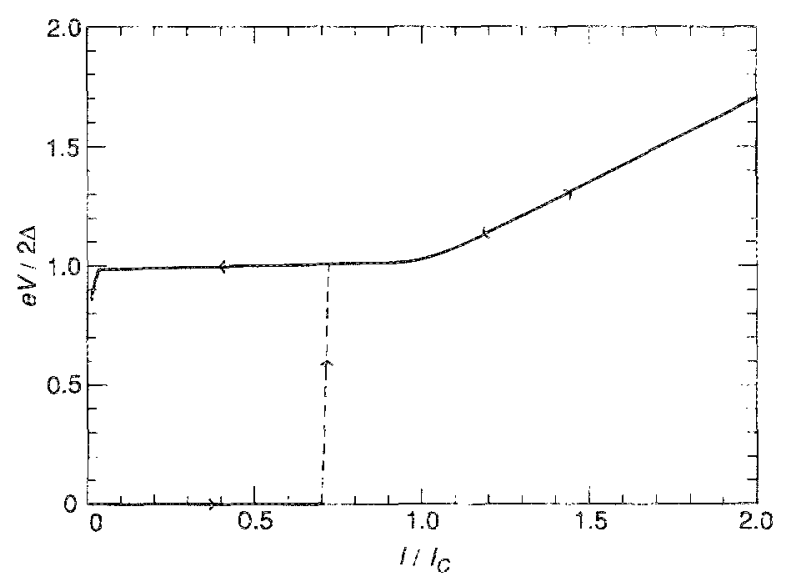

FIG. 3. $I-V$ curves obtained from time-domain simulations for a junction driven only by a de current source. The de current is ramped upward first and then downward. Here $\Delta / e=1.3 \mathrm{mV}, R_{N}=50 \Omega, C=100 \mathrm{fF}$, and $I_{C}=40 \mu \mathrm{A}$. large amount of hysteresis as would be expected for an $Z-Z$ curve with such a large value of capacitance and subgap leakage resistance $(\infty)$. Similar $I . V$ curves have also been computed for different values of the junction capacitance with reduced hysteresis for smaller values of the junction capacitance, as expected.

One might ask why the junction in Fig. 3 remained in the zero-voltage state unil only $75 \%$ of the critical current. One possibility would be that noise could decrease the critical current below the ideal value; however, a noise source was not added to this circuit. Although numerical "noise" inevitably exists due to numerical errors, especially discretization error, it does not appear to be very serious here. Rath$e r$, the initial conditions for the simulation, in particular the initial phase, are what actually determine the point at which the voltage jumps up. In the $I-D$ curves so far discussed, the initial plase was set at zero and a bias current was suddenly switched on. Since no quasiparticles flew at zero voltage and no pair currents flow at zero phase [recall $\left.l_{J}(t)=I_{C} \sin \phi\right]$, there is bias current $l_{\mathrm{dc}}$ entering the capacitor but not leaving it. Hence charge builds up, a voltage drop appears, and the finite voltage state may be entered. But suppose the initial phase is $\phi_{0}=\pi / 2$. Then, even at $I_{\mathrm{d}} \approx I_{C}$, the bias current entering the capacitor may be balanced by the pair currents, and the zero-voliage state may be the steady-state sohution. To test this, an $I-V$ curve was computed with the assumption that $\phi_{0}=\pi / 2$ and in this case the voltage jumped up at precisely the critical current, $40 \mu \mathrm{A}$. Numer:cal noise is not likely to be a major factor in the $I-V$ curves discussed here, because we have shown that it is not responsible for the junction prematurely entering the finite voltage stake.

Another important test, however, is the behavior of the simulation when the junction is driven by an ac current source. It is uscful to see if the Josephson step amplitudes generated by the simulation agree with the amplitudes predicted by the voltage-biased junction theory under the conditions in which zero-crossing steps are most expected. Simulations have been performed with the rf frequency $\omega_{L}=4 \Delta /$ h and with values of $\alpha=e V_{1} /$ Tho ranging from 0.4 to 1.2 . The initial voltage is set equal to the gap voltage $2 \Delta / e$ and the ac current source is turned on gradually. Signifcant bias current $\left(\sim 0.5 I_{c}\right)$ is supplied as the amplituce of the ac current source is ramped upward in order to maintain the junction in the finite voltage state. Then the bias current is ramped to a final value and it is observed whether phase lock can be maintained at that dc bias level. The following parameters were chosen: $C=500 \mathrm{fF}, R_{N}=50 \mathrm{\Omega}$, $2 \Delta / e=2.6 \mathrm{mV}, R_{l}=10 R_{N}$, and $\delta=0.02$. The results are presented in Table 1 , where they are compared with the theoretical prediction of the Werthamer theory. The step amplitudes from the simulations agree with those expected from the theory to a precision of about $1 \%-2 \%$, showing that the simulation is indeed correct.

\section{Numerical simulations for zero-crossing steps}

We now turn to the actual simulations for the zerocrossing steps. The initial voltage and phase were set at zero for all the simulations. The bias current was set at zero and 
TAELE I. Step amplitudes from simulations and Werthamer theory.

\begin{tabular}{ccc}
\hline & \multicolumn{1}{c}{$\begin{array}{c}\text { Full width of the first step } \\
\text { (Normalized to } I_{C} \text { ) }\end{array}$} \\
\cline { 2 - 3 }$a$ & Simulations & Werthamer theory \\
\hline 0.4 & 1.44 & 1.443 \\
0.5 & 1.72 & 1.739 \\
0.6 & 1.95 & 1.994 \\
0.7 & 2.16 & 2.204 \\
0.8 & 2.32 & 2.364 \\
0.9 & 2.43 & 2.470 \\
1.0 & 2.47 & 2.523 \\
1.1 & 2.49 & 2.521 \\
1.2 & 2.44 & 2.468 \\
\hline
\end{tabular}

${ }^{a} \omega_{L}=4 \Delta / n=7.9 \times 10^{12}, 2 \Delta / e=2.6 \mathrm{mV}, R_{N}=50 \Omega, R_{L}=10 R_{N}$, $\delta=0.02$.

${ }^{6}$ Theory assumes voltage bias at de and $\omega_{L}$, and calculation uses same $R_{N}$, $R_{\ell}, 2 \Delta / e, \omega_{L}$, and $\delta$ as the simulations.

the ac current source was turned on suddenly. In all cases $\Delta / e=1.3 \mathrm{mV}$ and $R_{N}=50 \Omega$. Four values for the parameter $\delta$ (discussed earlier and shown in Fig. 1) were chosen: 0 , $0.005,0.02$, and 0.05 . For $\delta=0.005$, a leakage resistance of $100 R_{N}$ was used; for $\delta=0.02$, two leakage resistances, $25 R_{N}$ and $10 R_{N}$, were used; and for $\delta=0.05$, only $25 R_{N}$ was used. For each case four values of capacitance were chosen: $20,50,200$, and $500 \mathrm{fF}$. These values of capacitance correspond to values of the parameter $\beta_{c}$ of $6.2,15.5,62$, 155 , respectively, where $\beta_{c} \equiv 2 e l_{C} R_{N}^{2} C / h^{2}$. The normalized frequency $\Omega$ becomes $\Omega=1.275$. For each capacitance ac current amplitudes were chosen producing values of $\alpha$ in the range of $0.4-1.2$. In these simulations $\Omega \geqslant \beta^{-1 / 2}$ and $i_{1} \gg 1$. It was observed that the waveform was always very nearly sinusoidal as expected from the RSJ model. Comparison between values of $\alpha$ found directly from the voltage waveform and from Eq. (58) show excellent agreement.

We observed many cases of zero-crossing steps. Especially encouraging is that they were observed even for the realistic $I-V$ curves, so that zero-crossing steps may be observable in real junctions at such high frequencies. The locking behavior for any particular set of runs differing only in values of $\alpha$ was visibly erratic.

One result is that in the time domain simulation, locking seems to peak at $\alpha \approx 0.5-0.6$ rather than at 0.8 , where one might expect from the voltage-biased theory calculations displayed in Figs. 2(a)-2(d). A possible explanation comes from the fact that the initial voltage was always set to zero and that the ac current source was turned on suddenly. The ac current source takes the form $I_{1} \sin \omega_{L} t$. Assuming that the ac current going into the capacitor is dominated by the ac current source and that by comparison the contribution from the Josephson effect is small, we obtain the voltage waveform from the formula $I=C d V / d t$. Thus a good approximation to $V(t)$ is $V_{0}-V_{L} \cos \omega_{L} t$, where $V_{L}=I_{1} / C \omega_{L}$ and $V_{0}=2 \Delta / e$. Thus $V(0)=V_{0}-V_{L}$, and by choosing this to be the input voltage at the start of the simulation, it is easiest for the junction to become phase locked to the first principal step. For $\alpha=0.5, V_{0}-V_{t}=0$, and so the initial voltage is ideal for phase locking. For $\alpha=1$ phase locking occurs less frequently even though the full step widths are greater, in part because the initial voltage is not close to the ideal value $-V_{0}$. Another factor in the reduced locking at $\alpha \approx 1$ appears to be that photon-assisted tunneling modifies the whole quasiparticle $I-V$ curve and can therefore Iift the steps away from the voltage axis for values of $\alpha$ near 1.0. Because the initial conditions here were not controlled as carefully as the simulations upon which Table $I$ is based, the locking ranges found were in general well below those expected from Figs. $2(a)-2(d)$.

It is also evident that zero-crossing steps are more easily achieved at smaller values of $\delta$, corresponding to higher values of the Riedel peak. This is expected, since Eq. (32a) for the Josephson step widths depends on $\tilde{I}_{J 1}$ which is proportional to $\ln (8 / \delta)$ at the rf frequency used. In addition, more often than not higher capacitance seems to increase the occurrence of zero-crossing steps. This is also expected, because at higher capacitance the ac Josephson current becomes smaller relative to the ac current source for fixed $\alpha$ and the voltage waveform becomes more nearly sinusoidal, which tends to increase phase locking.

Another point that deserves mention is that the response functions were truncated in the time domain in order to save computer time. This was feasible because the response functions die of in amplitude with time. For the pair currents greater rounding means a more rapid decrease in the amplitude of the time domain response function. Hence truncation of $\tilde{I}_{J 1}$ after a certain point in time has the effect of increasing the rounding of the Riedel peak in the frequency domain. This effect becomes more important for sharper peaks. The effective height of the Riedel peak was estimated by computing $\int_{0}^{\tau_{t}} I_{j}(t) \cos \omega t d t$, where $\tau_{c}$ is the cutof time, for $\omega=2 \Delta / \hbar$. The height was then converted into an equivalent value of $\delta$. For $\delta=0,0.005,0.02$, and 0.05 , the corresponding equivalent values were $\delta=0.015,0.015,0.028$, and 0.048 , respectively. We observe that for the sharpest peaks the errors are indeed greatest, while for the most rounded peak there appears not to be any appreciable error at all. The fact that the effective values of $\delta$ are the same for the cases $\delta=0$ and 0.005 helps to explain why the pattern of phase locking for the two cases appears similar. It should be kept in mind, however, that the efiect of rounding the peak through truncation is not necessarily the same as that of rounding it through the technique of $S e c$. II D, and the full significance of the equivalent values is not clear.

We conclude that there is general agreement between the voltage-bias theory and the more general time-domain simulations, which display a more complex behavior with increasing microwave drive level. It is important to realize the complex behavior of locking with $\alpha$ that we have described may be due to problems with initial conditions, since these simulations were done only for one set of initial conditions, namely $\phi(t)=V(t)=0$ for $t<0$. Better agreement with voltage-bias theory (Table I) was obtained only after a larger exploration of initial conditions than was performed for the study of the locking range with $\alpha$. However, the results we have obtained are quite tantalizing and merit further time-domain simulations. Our simulations generally sup- 
port the conclusions based on the analytical and numerical frequency-domain calculations.

\section{SUMMARY AND CONGLUSIONS}

We have shown from the Werthamer theory that zerocrossing steps are possible in principle at high frequencies as long as the voltage waveform is nearly sinusoidal. Limits on the amount of permitted rounding of the Riedel peak were derived from the analytical calculations of Secs. II D and II E. Numerical frequency-and time-domain computations for realistic junctions support these limits. We have observed that in the time domain simulations the capacitance was surficiently large to ensure that the voltage waveform was very rearly sinusoidal. This leaves us with the issue of the stability of these steps, and further numerical analysis and simulations may be necessary to help address this question. Our results show that zero-crossing steps do in some cases appear to be stable for realistic junctions, at least for the short periods of time over which the simulations were run. A number of points should be kept in mind, however. First, the erratic locking patterns observed suggest that we are near the edges of the steps at zero bias current for our set of initial conditions. These are likely to be the least stable places on the steps. Second, there was no source of noise other than numerical noise, which does not seem to have been very important in these simulations. Real junctions, however, will always contain intrinsic noise that can disrupt phase lock. Analysis of thermal and shot noise effects on the phase-locking behavior suggests that stability of the phase lock should be significantly better at high frequencies than at the frequencies currertly used for voltage standards. Third, it should also be mentioned that in the simulations the convolution integral was cut of beyond a certain point. The response functions die of with time, and at trmes at which the amplitudes are sufficiently diminished, the contribution to the rotal integral may be neglected. While this saves enormously in computer time, sone error inevitably results. The effect of cutting of the response functions at later times in the time domain is essentially that of increasing the rounding of the Riedel peak in the frequency domain. This may affect our results somewhat, although the effect should be small here. Analysis of the practical considerations involved in making a standard at high frequencies shows that optimum junction current density, length, width, and microwave rower levels are all within reasonable limits.

We have also shown that the Riedel peak can increase the width of the Josephson steps at the gap voltage when the microwave drive frequency is a submultiple of twice the gap frequency. This may have a practical benefit since it is easier to find oscillators with sufficiently large power levels and tunability at 400 and $600 \mathrm{GHz}$ as compared to $1.2 \mathrm{THz}$ ( $4 \Delta / h$ for $\mathrm{Nb} / \mathrm{PbInA}$ u edge junctions). Sufficient power levels for such frequencies could be achieved by harmonic multiplication of lower-frequency $(100-\mathrm{GH} z$ range) Gunn oscillator frequencies.

It is clear from analytical and numerical computations and the simulations done so far that the best conditions under which to search for zero-crossing steps occur when the Riedel peak is sharpest, so it is clear that junction quality is an important factor. In addition, the capacitance should be large enough to ensure that the voltage is sinusoidal. ac current sources must have sufficient amplitude for $\alpha \approx 0.5$ for junctions driven at twice the gap frequency. Subharmonically driven junctions need somewhat higher values of $\alpha$ for maximum step amplitude. Further simulations or experiments would be useful, and it would probably be best to focus on these conditions. However, previous arguments that zero-crossing steps could probably never be observed above half the gap voltage are incorrect due to neglect of the Riedel peak. Both our theoretical calculations and numerical simulations indicate that such steps can indeed occur up to the gap voltage.

\section{ACKNOWLEDGMENTS}

We are pleased to thank P. A. Jaminet for a critical reading of the manuscript, as well as W. Glaser, who wrote a preliminary version of the FORTRAN code for the simulations. Initial computer time for this work was supported in part by a grant from BBM. One of the authors (W.C.D.) acknowledges partial support through the L. W. Frohlich Research Fellowship of the New York Academy of Sciences. Work on superconducting tunnel junctions at the Space Sciences Laboratory is supported in part by the U.S. Army Research Office under Contract No. DAAG29-85*K-0254.

'B. D. Josephson, Phys. Lett. 1, 251 (1962); S. Shapiro, A. R. Janus, and S. Holly, Rev. Mod. Phys. 36, 223 (1964).

${ }^{2} M$. T. Levinsen, R. Y. Chaio, M. J. Feldman, and B. A. Tucker, Appl. Phys. Lett. 31, 776 (1977).

${ }^{3}$ R. L. Kautz, Appl. Phys. Lett. 36, 386 (1980).

4. Niemeyer, I. H. Hinken, and R. L. Kautz. Appl. Phys. Letr. 45, 478 (1984).

${ }^{5 F}$. L. Eloyd, C. A. Hamition, J. A. Beall, D. Go, R. H. Ono, and R. E. Harris, IEEE Electron Dev. Lett. EDL-8, 449 (1987).

${ }^{6} \mathrm{~W} . \mathrm{C}$. Stewart, Appl Phys. Lett. 12, 277 (1968); D. E. McCumber, J. Appl. Phys. 39, 3113 (1968).

'C. A. Mamilton, Fhys. Rev. B 5, 912 (1972).

${ }^{8}$ D. G. McDonald. A. S. Risley, J. D. Cupp, K. M. Evenson, and J. R. Ashley, Appl. Phys. Lett. 20, 296 (1972).

'D. A. Weitz, W. J. Skocpol, and M. Tinkham, Phys. Rev. B 18,3282 (1978).

${ }^{10}$ W. C. Danchi, F. Habbal, and M. Tinkham, Appl. Phys. Lett. 41, 883 (1982).

"F. Habbal, W. C. Danchi, and M. Tinkham, Appl. Phys. Lett, 42, 296 (1983).

II. R. Werthamer, Phys. Rev. 147, 255 (1966).

1.A. H. Dayem and R. J. Martin, Phys. Rev. Lett. 8, 246 (1962).

${ }^{14}$ P. K. Tien and J. R. Gordon, Phys. Rev. 129, 647 (1963).

isE. Riedel, Z. Naturforsch. 19A, 1634 (1964).

10R. E. Harris, Phys. Rev. B1B, 3329 (1975); Phys. Rev, B 13.3818 (1976).

${ }^{17}$ R. E. Harris, J. Appl. Phys. 48, 5188 (1977).

:R. L. Kautz, J. Appl]. Phys. 52, 3528 (1981).

${ }^{19}$ S. A. Buckner and D. N. Langenberg, J. Low Temp. Phys. 22, 569 (1976).

${ }^{20}$ A. B. Zorin, K. X. Likharev, and S. I. Turovers, IEEE Trans. Magn. MAG-19,629 (1983).

${ }^{2 !}$ R. E. Harris, Phys. Rev. B 10, 84 (1974).

"2'Sce, for example, J. R. Tucker and M. J. Feldman, Rev. Mod. Phys. 57, 1055 (1985).

${ }^{23}$ W. C. Danchi, J. Bindslev-Hansen, M. Octavio, F. Habbal, and M. Tinkham, Phys. Rev. B 30, 2503 (1984).

${ }^{24}$ R. L. Kautz, C. A. Hamiton, and F. L. Lloyd, IEEE Trans. Magn. MAG. 23, $883(1987)$.

${ }^{25}$ R. L. Kautz, J. Appl. Phys. 52, 3528 (1981); 52, 6241 (1981).

${ }^{26} \mathrm{Q}$. Hu, J. U. Free, M. Tansiti, O. Liengme, and M. Tinkham, IEEE Trans. Magn. MAG.21, 590 (1985). 
${ }^{27}$ J. H. Magerlein, IEEE Trans. Magn. MAG-17, 286 (1981).

${ }^{28}$ M. J. Wengler, D. P. Woody, R. E. Miller, and T. G. Phillips, int. J. Infrared and Millimeter Waves 6, 697 (1985).

${ }^{29}$ K. E. Irwin, T. Van Duzer, and S. E. Schwartz, IEEE Trans. Magn. MAG-21, 216 (1985).
${ }^{30}$ T. C. G. L. Sollner and S. D. Powell, IEEE Trans. Magn. MAG-19, 605 (1983).

${ }^{36}$ W. C. Danchi and E. C. Sutton, J. Appl. Phys. 60, 3967 (1986).

${ }^{32}$ S. D. Conte and C. deBoor, Elementary Numerical Analysis (McGrawHi11, New York, 1980), pp. 95-99. 